\title{
Modeling the warm absorber in active galactic nuclei
}

\author{
A. Różańska ${ }^{1}$, R. Goosmann ${ }^{2}$, A.-M. Dumont ${ }^{2}$, and B. Czerny ${ }^{1}$ \\ 1 Copernicus Astronomical Center, Bartycka 18, 00-716 Warsaw, Poland \\ e-mail: agata@camk.edu.pl \\ 2 Observatoire de Paris-Meudon, LUTH, Meudon, France
}

Received 18 January 2005 / Accepted 31 January 2006

\section{ABSTRACT}

\begin{abstract}
We present a wide grid of models for the structure and transmission properties of warm absorbers in active galactic nuclei (AGN). Contrary to commonly used constant density models, our absorbing cloud is assumed to be under constant total (gas plus radiation) pressure. This assumption implies the coexistence of material at different temperatures and ionization states, which is a natural consequence of pressure and thermal equilibrium. Our photoionization code allows us to compute the profiles of the density, the temperature, the gas pressure, the radiation pressure and the ionization state across the cloud, and to calculate the radiative transfer of continuum and lines including Compton scattering. Therefore, equivalent widths of both saturated and unsaturated lines are properly modeled. For each pair of the incident spectrum slope and the ionization parameter at the cloud surface there is a natural upper limit to the total column densities of the cloud due to thermal instabilities. These maximum values are comparable to the observational constraints on the column density of warm absorbers which may give support to constant total pressure models. In all models we note considerable absorption around $6.4 \mathrm{keV}$ which modifies the intrinsic relativistically broadened iron line profile originating in an accretion disk illuminated atmosphere. Our models can be applied to fitting the spectroscopic data from the XMM-Newton and Chandra satellites.
\end{abstract}

Key words. radiative transfer - line: identification - galaxies: Seyfert - X-rays: galaxies - galaxies: active

\section{Introduction}

The first X-ray absorption feature due to ionized heavy elements was recognized by Halpern (1984) in the X-ray spectrum of the Sy1 galaxy MR 2251-178 observed by the EINSTEIN satellite. The author related the observed jump in flux around $1 \mathrm{keV}$ to the absorption edge of OVIII. Therefore, as Halpern concluded, $\mathrm{X}$-rays emitted from the central region of an active galaxy traveling toward an observer encounter a "warm absorber" - material with an electron temperature lower than the temperature of collisionally ionized gas with a similar level of ionization.

Results from EXOSAT, ROSAT, GINGA, ASCA, and Beppo-SAX satellites showed that warm absorbers were common among Seyfert galaxies. Nandra \& Pounds (1994) have suggested that more than $50 \%$ of Sy1s contain a warm absorber, and later results confirmed such occurrence rate (e.g. Reynolds 1997; George et al. 1998). Those conclusions were based exclusively on the detection of absorption edges (for instance in MCG-6-30-15, Nandra \& Pounds 1992). The detector areas and the spectral resolution of those satellites did not allow us to see any absorption lines from highly ionized species.

The situation changed since 1999, when large X-ray telescopes Chandra and XMM-Newton started to operate, with their $\mathrm{X}$-ray grating instruments working in the energy range up to almost $10 \mathrm{keV}$. Several tens of absorption or emission lines were observed and identified in NGC 3783 (Kaspi et al. 2002; Behar et al. 2003; Netzer et al. 2003; Krongold et al. 2003), NGC 5548 (Kaastra et al. 2002), NGC 1068 (Kinkhabwala et al. 2002), NGC 7469 (Blustin et al. 2003), MCG-6-30-15 (Turner et al. 2004). In other objects the number of fitted lines is lower and/or their detection is less firm, but the results still strongly support the presence of a warm absorber in those Seyferts (NGC 4051, Collinge et al. 2001; Mrk 509, Yaqoob et al. 2003; TonS180, Różańska et al. 2004). Warm absorber lines were even detected in a distant blazar at $z=4.4$ (Worsley et al. 2004).

Detected lines are basically consistent with the unification scheme of AGN based on the presence of the dusty/molecular torus. According to that scheme, Sy1 are objects seen face-on and Sy2 are objects seen edge-on (Antonucci \& Miller 1985; for application to X-ray band see Mushotzky et al. 1993). The major characteristic of the X-ray spectra of Sy2 galaxies is that they are strongly absorbed at low energies, and that their emission lines, especially the iron $\mathrm{K} \alpha$ line, have large equivalent widths (Turner et al. 1997) since their intensity is measured with respect to the heavily obscured direct continuum (Weaver \& Reynolds 1998) or only with respect to the scattered continuum (in Compton-thick objects), as discussed by Bassani et al. (1999). In some cases of Compton-thin Sy2 obscuration may be due to the host galaxy and unrelated to disk/torus orientation (Matt 2000; Guainazzi et al. 2001) The spectra of Sy1 galaxies are predominantly featureless, show little low energy absorption, and the equivalent width of the iron $\mathrm{K} \alpha$ line is much lower than in Sy2. Some Sy1 show relativistically broadened iron $\mathrm{K} \alpha$ line profiles which additionally supports the view that in Sy1 we directly observe the innermost part of the nucleus. The scattering medium may be identical to the warm absorber and we have side view of this medium in Sy2 galaxies while we see the nucleus through it in Sy1. Indeed, X-ray spectra of some Sy2 galaxies show many narrow emission lines, (e.g. NGC 1068, Kinkhabwala et al. 2002; NGC 4507, Matt et al. 2004; Mkn 3, Pounds \& Page 2005; NGC 4151, Schurch et al. 2004) indicating that ionized material may extend beyond the shielding 
torus. In Sy1 spectra mostly absorption lines are observed (e.g. NGC 3783, Kaspi et al. 2002: NGC 5548, Kaastra et al. 2002; Mrk 509, Yaqoob et al. 2003) without strong absorption of continuum, suggesting that in those objects we see directly the nucleus through the ionized plasma. Some contribution of emission lines in Sy1 X-ray spectra is also expected (e.g. Netzer 1993; Collin et al. 2004) and seen in the data (e.g. NGC 3783, Behar et al. 2003; NGC 7469, Scott et al. 2005). However, the constraints from Sy2 galaxies for the ionized medium cannot be used directly to Sy1 galaxies since in Sy2 galaxies we may observe only the outer part of the plasma distribution, due to the obscuration by the torus, while warm absorber features may come predominantly from the inner part.

A majority of these absorption lines shows a velocity shift of the order of a few hundreds $\mathrm{km} \mathrm{s}^{-1}$ (Kaspi et al. 2001; Kaastra et al. 2002) suggesting that the warm absorber is outflowing. A strong high velocity outflow was recently reported in several objects, mostly radio-quiet quasars (e.g. $60000 \mathrm{~km} \mathrm{~s}^{-1}$ and $120000 \mathrm{~km} \mathrm{~s}^{-1}$ in APM 08279+5255, Chartas et al. 2002; $23000 \mathrm{~km} \mathrm{~s}^{-1}$ in PG1211+143, Pounds et al. 2003a; $63000 \mathrm{~km} \mathrm{~s}^{-1}$ in PG0844+349, Pounds et al. 2003b, $30000 \mathrm{~km} \mathrm{~s}^{-1}$ in IRAS 13197-1627, Dadina \& Cappi 2004; $26000 \mathrm{~km} \mathrm{~s}^{-1}$ in PG 1404+226, Dasgupta et al. 2005). However, the results are based on a few detected lines, so line identifications and, consequently, the determined high outflow velocities can be questioned (Kaspi 2004). The origin of the outflow and its geometry is still under discussion (Crenshaw et al. 2003a; Blustin et al. 2005).

It is more difficult to estimate the radial distance of the warm absorber from the nucleus. Absorption lines most probably form somewhere between the broad line region (BLR) and the narrow line region (NLR) (i.e. from about 0.01-0.1 up to 10 pc from nucleus; see Crenshaw et al. 2003a; Blustin et al. 2005). Variability in the overall warm absorber properties was reported for a few sources (MR 2251-178, Halpern 1984; Kaspi et al. 2004; MCG-6-30-15, Reynolds et al. 1995; H1419+480, Barcons et al. 2003; NGC 4395, Shih et al. 2003; NGC 3516, Netzer et al. 2002). Netzer et al. (2002) and Barcons et al. (2003) concluded that the changes observed are consistent with varying ionization of the gas. The lack of short-timescale (days) response of the warm absorber to the change of the continuum can be used to put lower limits to the warm absorber distance (e.g. 0.5-2.8 pc for NGC 3783, Behar et al. 2003; similar limits were given by Netzer et al. 2003). Krongold et al. (2005) detected a response of the warm absorber to the changes of the continuum on a timescale of 31 days in NGC 3783, deriving an upper limit of $6 \mathrm{pc}$ for the distance of the warm absorber. The shortest variability timescale of $\sim 10^{4} \mathrm{~s}$ has been detected in the warm absorber in MCG-6-30-15 (Otani et al. 1996; see also Turner et al. 2004) locating the plasma responsible for the OVIII edge within the distance of $10^{17} \mathrm{~cm}$ from the nucleus. The spectral analysis of this source indicates that the highly ionized warm absorber is dust-free, with dust contribution in this source coming from a distant zone, hundreds of pc from the nucleus (Ballantyne et al. 2003).

The same medium is most probably responsible for narrow absorption lines seen in the UV spectra of many AGN (for a review, see Crenshaw et al. 2003a). Some kinematic components discovered in UV coincide with those discovered in soft X-rays but for other components no such correspondence is seen (e.g. Behar et al. 2003 for NGC 3783, Crenshaw et al. 2003b for NGC 5548, Kaspi et al. 2004 for MR 2251-178; Scott et al. 2005 for NGC 7469; Gabel et al. 2005 for NGC 3783). However, the resolving power of the $\mathrm{UV}$ observations is high $(R \sim 20000)$ while X-ray data resolution is much lower $(R \sim 1000)$, which makes the comparison difficult, as discussed by Crenshaw et al. (2003a). Analysis usually suggests that UV and X-ray absorption features are consistent with arising in the same gas, but with stratified ionization (e.g. Barcons et al. 2003; Kaspi et al. 2004; Scott et al. 2005; Gabel et al. 2005). Since the absorption features appear in the profiles of the broad emission lines like CIV, and are occasionally deep, this serves as an argument that the absorbing region is located outside the BLR.

The column density of the warm absorber is generally estimated to be about $10^{21-23} \mathrm{~cm}^{-2}$, and absorbing gas contains heavy elements mostly in the form of helium- and hydrogen-like ions. However, accurate measurements of the column density are quite complex. Most determinations are based on detection of absorption edges, but in some cases edges are undetectable while lines are clearly seen (Kaastra et al. 2002; Różańska et al. 2004). Also the estimates of column densities from edges do not always confirm estimates derived from the absorption line analysis (Kaspi et al. 2002).

The ionization state of the gas required to reproduce the observed absorption or emission lines seems to be quite complex. In many objects, predictions based on a single cloud at one specific ionization state cannot explain the data, so an absorbing material is modeled using at least two photoionization regions, which are required to explain the presence of lines from matter in different ionization states (see in the case of Sy1: NGC 4051, Collinge et al. 2001; NGC 5548, Kaastra et al. 2002; NGC 3783, Kaspi et al. 2002; Netzer et al. 2003 and Krongold et al. 2005; H0557-385, Ashton et al. 2006). The same conclusion was drawn from fitting of the absorbed continuum (strongly absorbed Sy1: Mkn 304, Piconcelli et al. 2004; IC 4329A, Steenbrugge et al. 2005a,b; in the case of a dwarf galaxy with an active nucleus: NGC 4395, Shih et al. 2003; and in case of Sy2: NGC 4507, Matt et al. 2004).

The idea of the warm absorber being under constant pressure was developed even before the Chandra and XMM-Newton satellites were launched (Netzer 1993; Krolik \& Kriss 1995; Netzer 1996). It is well known that cold/warm material irradiated by hard X-rays should be strongly stratified, and eventual thermal instabilities lead to its clumping (Krolik et al. 1981). If thermal instabilities are strong, none of the existing photoionization codes can describe the unstable zone. This is because radiative transfer codes are based on unique density and temperature profiles, and do not accept situations where for one value of optical depth the solution gives three different values of temperature and density.

Therefore many models of the warm absorber under constant pressure assume that those two discrete phases already exist. Usually two or three zones at different constant densities are assumed to have the same dynamical ionization parameter, $\Xi$, which is the ratio of ionization pressure to the gas pressure (Krolik et al. 1981). In such a situation, calculations of transfer of X-ray radiation through two constant density zones are done separately and then spectrum is merged together depending on the covering factor (Netzer 1993).

In this paper we study the situation where X-ray radiation is no so hard and strong that separation into two phases takes place. Instead, our warm absorber is strongly stratified and radiation passes through different densities and ionization stages. This situation was considered theoretically by Krolik \& Kriss (2001), Krolik (2002). The stratification of our warm absorber is determined physically by radiative properties, and the only assumption which we make is constant total pressure within a cloud. We use the photoionization code TITAN developed by 
Dumont et al. (2000) (see Dumont et al. 2003 for implementation of the Accelerated Lambda Iteration method) to compute the synthetic spectra for a systematic set of model parameters. The advantage of our calculations is that we compute the full radiative transfer of continuum and lines what allows for proper treatemet of saturated lines. Recent observations suggest that saturated lines are often present in a warm absorber (Kaspi et al. 2002; Różańska et al. 2004). The transfer is done in the stratified medium, and the density profile is determined self-consistently with the radiation transfer to fulfill the condition of pressure equilibrium across the cloud. We aim to understand these AGN that show clear absorption lines in their Chandra or XMM spectra so that the warm absorber is located in the line of sight to the observer. We concentrate on modeling the transmission spectra and absorption lines and therefore our models can be used for Sy1 galaxies.

We describe our model in Sects. 2 and 3, results are presented in Sects. 4-6. Section 7 compares our models to observations and Sect. 8 contains concluding remarks.

\section{Description of the model}

The main assumption of our model is that the structure of a single warm absorber cloud is determined by the condition of constant total, $P_{\text {gas }}+P_{\text {rad }}$, pressure. The value of this pressure is self-consistently determined by solving non-LTE ionization equilibrium, thermal equilibrium and radiative transfer throughout the cloud, thus providing a density and ionization profile inside a cloud.

We model an irradiated cloud using a plane parallel approximation. The total column density of the cloud, $N_{\mathrm{H}}$, is a free parameter in our model.

The second model parameter is the ionization parameter, $\xi$, determined at the cloud surface

$\xi=\frac{L}{n_{0} R^{2}}=\frac{4 \pi F}{n_{0}}$,

where $L$ is the luminosity of the central source, $n_{0}$ is the number density at the cloud surface, at its illuminated side, and $R$ is the distance of the cloud from the central source. There is no difference if we use a small $\xi$ or a dynamical large $\Xi$ ionization parameter which is defined as $\Xi=F /\left(c P_{\text {gas }}\right)$ since both parameters are used only to specify the amount of X-ray flux illuminating the cloud surface. In our models both ionization parameters change with the optical depth due to the change in the density and in the irradiating flux, and this stratification is computed self consistently.

The X-ray illuminating continuum is assumed to have a power-law shape, as it is usually observed in many Seyferts, and the power-law photon index, $\Gamma$, is a model parameter.

The photoionization code TITAN developed by Dumont et al. (2000) solves the full radiative transfer of the ionizing continuum in a two stream approximation. The transfer method for the lines and continuum used in this code has been modified to use the accelerated lambda iteration (ALI) method, which is especially well suited to rapid convergence in the cores of lines which may be optically thick (Dumont et al. 2003). The computations are done assuming complete redistribution function in the lines. Partial redistribution is mimicked by a Doppler profile for some of the most intense resonant lines.

The comparison of proper ALI computations with escape probability approximation was shown by Dumont et al. (2003) and Collin et al. (2004). In the case of emission lines the errors in the line fluxes are typically of the order of $30 \%$ for column densities $10^{20} \mathrm{~cm}^{-2}$ and a factor of five for column densities of $10^{23} \mathrm{~cm}^{-2}$, compared to calculations using the escape probability formalism.

TITAN includes all relevant physical processes from each ion level. The population of each level is computed solving the set of ionization equations coupled with the set of statistical equations describing the excitation equilibrium. However, some low ionization states are more approximately treated.

The following processes are taken into account: radiative and collisional ionization in all levels, recombinations in all levels, and radiative and collisional excitations and de-excitations for all transitions.

We consider ten main heavy elements including iron. TITAN transfers "only" 900 lines while some photoionization codes (XSTAR, CLOUDY) collect over 4000 lines or more. On the other hand, TITAN is also designed to calculate radiative transfer in Compton thick media, which means that we are able to compute transfer of optically thick lines through any stratified cloud, and also through an atmosphere in hydrostatic equilibrium (Różańska et al. 2002). The same code is applicable for a whole range of column densities (from small to large), and a whole range of ionization states.

The Compton heating/cooling balance through the cloud is taken into account since for a hard photon index, $\Gamma=1.5$, Compton heating is comparable to photoionization heating. The Compton balance of photons up to $26 \mathrm{keV}$ is computed in TITAN while the Compton balance of photons between 26 up to $100 \mathrm{keV}$ is computed using code NOAR (Dumont et al. 2000). The NOAR is a radiative transfer code, based on the Monte Carlo method, to compute a Compton heating profile for a plane stratified atmosphere. This Compton heating/cooling profile is read by TITAN and taken into consideration solving the radiative transfer. Usually three iterations between NOAR and TITAN are enough to determine the Compton heating/cooling curve. For the sets of our models with the same $\Gamma$ the Compton heating does not vary much with $\xi$ and $N_{\mathrm{H}_{\mathrm{tot}}}$ of the warm absorber. Hence, we only derived a few representative Compton profiles, for different $\Gamma$ and we adopted them to all other models to perform our computations. Comptonization of line photons is included.

Generally our code models the reflected and outward spectrum from the slab with the assumption that both are emitted isotropically. In Fig. 1 we present all spectra available for one particular cloud. The advantage of our code is that we do not separate absorbed and emitted spectra which are derived from two different parts of the solution of radiative transfer. This procedure is done in many previous attempts starting from Netzer (1993). In TITAN we solve transfer of absorption and emission continuum and lines simultaneously producing the outward spectrum (see Fig. 1), which is a mixture of absorption and emission features. Nevertheless, if a warm absorber cloud is on the line of sight to the observer, we see the outward cloud spectrum emitted only in the direction normal to the slab, i.e. pointing to the observer.

In this first paper we examine only the absorption features, therefore the final spectra presented below are purely absorption spectra, i.e., outward in the direction toward the observer (transmitted). This is equivalent to the situation where the covering factor of the warm absorber is small, but a single warm absorber cloud is large and located on the line of sight to the observer. The model is applicable to Seyfert 1 galaxies since in those objects we observe mostly absorption lines. Although the absorbed spectra do not contain any emission lines, the temperature and ionization structure were computed with full transfer 


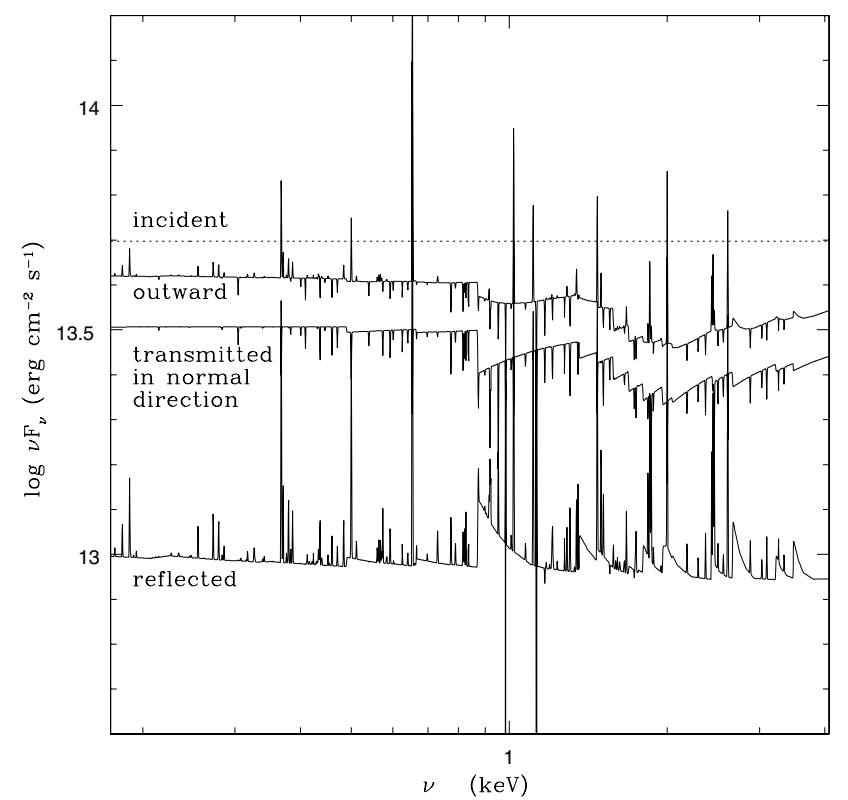

Fig. 1. All spectra available from one considered cloud $\left(\xi=10^{5}\right.$, $\left.N_{\text {tot }}=10^{24}\right)$. The incident power-law is indicated by the dotted line and is assumed to be isotropic. The reflected spectrum is emitted isotropically on the same side of the cloud, while the outward spectrum is emitted isotropically on the opposite side of the cloud. Transmitted spectrum is the outward spectrum in the normal direction to the observer.

of absorbed, emitted and reflected radiation. Reflected and outward spectra in other directions will be treated more carefully in the next paper. UV lines in all models are very weak since the illuminated continuum starts from $0.01 \mathrm{keV}$, however our code calclulates most of important the UV transitions.

In this paper we discuss a grid of models with no turbulent velocities to see the main trends in the properties of the absorbing matter. But we also present two models with non-zero turbulent velocity, the same for all ions, to trace how it affects final equivalent widths of lines (see also a special case considered in Różańska et al. 2004).

\section{Grid of parameters and numerical limitation}

All computations are done assuming that the surface of a warm absorber has a number density $n_{0}=10^{11} \mathrm{~cm}^{-3}$. We have checked that even a much lower value of the surface density does not change the cloud structure and its absorbed spectrum. Results are the same down to $n_{0}=10^{5} \mathrm{~cm}^{-3}$. This degeneracy does not allow us to determine the distance to the warm absorber since from observed spectra we do not have any constraints on the surface density of the medium. The ionization parameter $\xi$ gives direct information on the flux of the ionizing radiation that hits the surface of the warm absorber. In our model we vary $\xi$ from $10^{3}$ to $10^{5} \mathrm{erg} \mathrm{cm} \mathrm{s}^{-1}$.

We assume that the ionizing continuum comes from the center of an active nucleus and has a power law shape. The photon index varies from 1.5 to 2.5 as observed for the hard X-ray band. The illuminating X-ray continuum in all models extends from $0.01 \mathrm{keV}$ to $100 \mathrm{keV}$.

When computing warm absorber models under constant pressure we deal with the well known problem of an illuminated atmosphere with heavy elements. If the incident spectrum is hard enough and/or the abundance of heavy elements is solar or higher, a thermal instability develops (Fields 1965;

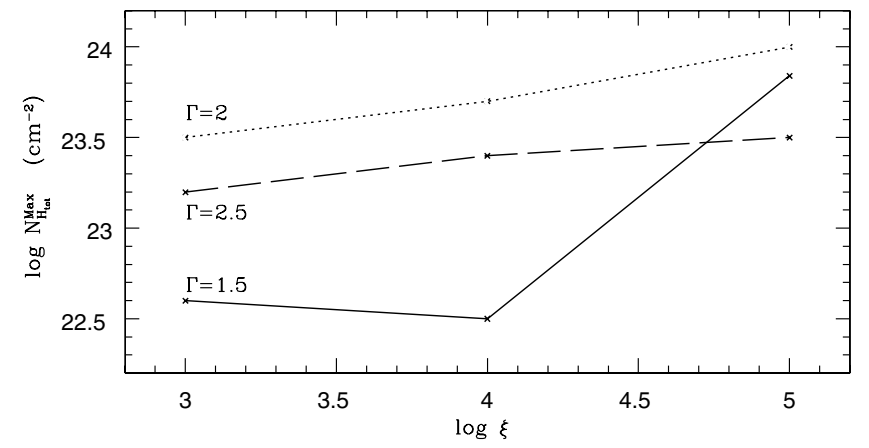

Fig. 2. The maximum total column density versus ionization parameter for each photon index. For a given value of $\Gamma$ and $\xi$ the structure of clouds with column densities higher than $N_{\mathrm{H}_{\mathrm{tot}}}^{\mathrm{Max}}$ cannot be determined because of thermal instabilities.

Krolik et al. 1981) which affects the conditions in the surface layers of the irradiated stellar or accretion disk atmospheres, including AGN disks (e.g. Różańska \& Czerny 1996).

The instability occurs when for a single value of the dynamical ionization parameter $\Xi=F_{\text {tot }} / c P_{\text {gas }}$ the energy balance equation has three solutions with different temperatures and densities. Therefore, in a constant density medium it is not possible to obtain any instabilities but they develop if the density of the medium can adjust to the local conditions. Among these three solutions, one is unstable, and two others are stable, but there is no stable solution that is continuous through the whole irradiated layer. In our case we assume constant pressure instead of constant density and we may expect thermal instabilities in the clouds with a total column density high enough for the temperature to drop considerably inside the cloud (see Sect. 4).

Generally, for each set of $\Gamma$ and $\xi$ we consider a range of values for the logarithm of the column density, $\log N_{\mathrm{H}}$, starting with 21. Whenever possible, we extended our grid to $\log N_{\mathrm{H}}=$ 23.5. However, for specific values of the initial ionization parameter and/or steepness of X-ray spectrum we were not able to achieve thermal equilibrium. There is a maximum total column density, $N_{\mathrm{H}_{\mathrm{tot}}}^{\mathrm{Max}}$, for which instabilities are so strong that computations failed. For this reason our grid of total column densities is not the same for each set of $\Gamma$ and $\xi$. These maximum values of the total column density are shown in Fig. 2 for all combinations of $\Gamma$ and $\xi$.

The maximum value $N_{\mathrm{H}_{\mathrm{tot}}}^{\mathrm{Max}}$ rises with an increase of the ionization parameter but the relation is not strictly monotonic, and the dependence on the spectral slope is even more complex. The most difficult case was $\Gamma=1.5$ and $\xi=10^{4}$, where a temperature fall appeared even for low total column densities, and we were able to find a stable cloud only up to the $N_{\mathrm{H}_{\mathrm{tot}}}=3.16 \times$ $10^{22} \mathrm{~cm}^{-2}$. Under those special conditions, line cooling due to a single iron ion strongly dominated all other cooling mechanisms, and the medium was optically thick in the emitted lines. Therefore, the local temperature strongly depended on the presence or absence of a layer behind the considered zone. The cooling of the outer zone served as a heating mechanism to the inner zone, compensating exactly the losses. As a result, the Thomson depth of the hot zone depended roughly linearly on the assumed $N_{\mathrm{H}_{\mathrm{tot}}}$.

In this paper we also study the case when absorbing matter has a turbulent velocity. For this purpose we consider two cases, one with $v_{\text {turb }}=100 \mathrm{~km} \mathrm{~s}^{-1}$, and one with $v_{\text {turb }}=300 \mathrm{~km} \mathrm{~s}^{-1}$, the same for each ion. 


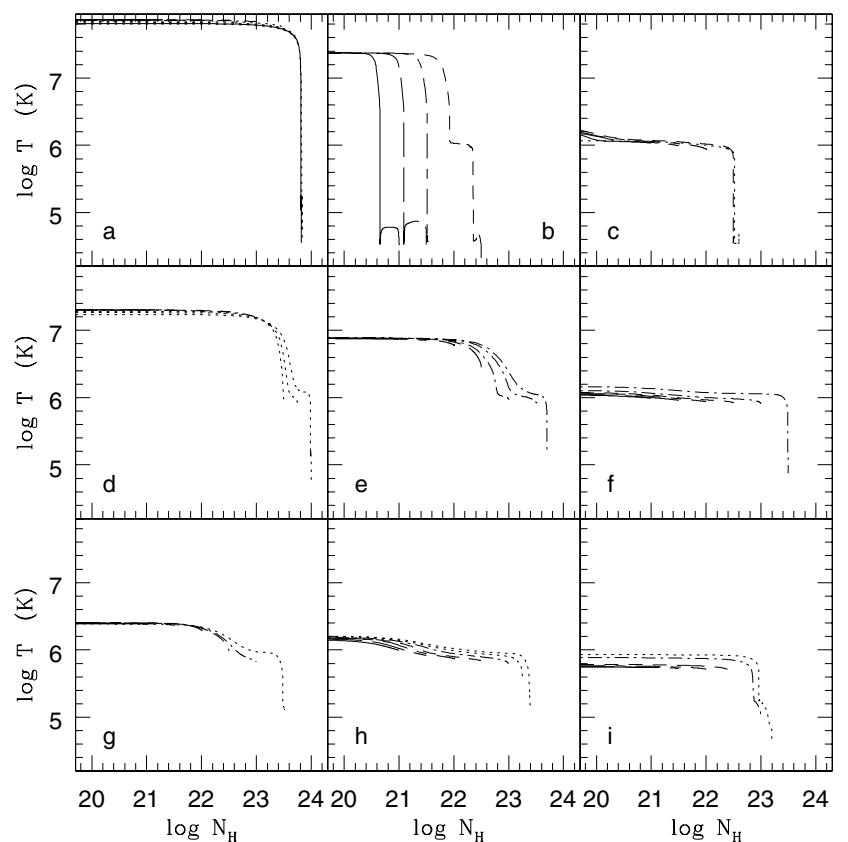

Fig. 3. The temperature profiles within the irradiated clouds at constant pressure. The different panels show the dependence on the total column density, starting with $\log N_{\mathrm{H}_{\text {tot }}}=21$, with step sizes of 0.5 . The step size decreases to 0.1 when approaching the value of $\log N_{\mathrm{H}_{\mathrm{tot}}}^{\mathrm{Max}}$. Panels a), b), c) show the results for $\xi=10^{5}, 10^{4}$ and $10^{3}$, and for $\Gamma=1.5$. Panels $\mathbf{d}$ ), e), f) show respectively the same ionization parameters but for $\Gamma=2$. The last three panels, $\mathbf{g}), \mathbf{h}), \mathbf{i})$, present the cases for $\Gamma=2.5$.

\section{Structure of the warm absorber}

We expect that at the illuminated face of a cloud the temperature will be high and density low, and a high temperature equilibrium on the "S curve" (Krolik et al. 1981) will be reached. This layer is expected to be optically thin despite some lines which may be saturated. Deeper inside the warm absorber the illuminating continuum will be absorbed by heavy elements and we expect that the temperature structure will show a strong decrease with accompanying increase of the density, while the ionization state decreases, but not so rapidly. Therefore after this geometrically extended optically thin layer, we expect geometrically thin layers with strong temperature and density gradients becoming optically thicker.

The temperature structures of all computed clouds are given in Fig. 3. Models with $\Gamma=1.5$ are presented in panels $a, b, c$ for $\xi=10^{5}, 10^{4}$ and $10^{3}$ respectively. The same sequence of $\xi$, but for $\Gamma=2$, is shown in panels $\mathrm{d}$, e, $\mathrm{f}$, and the softest intrinsic spectrum with photon index 2.5 is presented in panels $\mathrm{g}, \mathrm{h}$ and $\mathrm{i}$. The density structure for the same set of models is presented in Fig. 4.

For each set of $\Gamma$ and $\xi$ we present results for a grid of column densities starting from $N_{\mathrm{H}_{\text {tot }}}=10^{21}$ up to about $N_{\mathrm{H}_{\mathrm{tot}}}^{\mathrm{Max}}$, depending on the appearance of thermal instabilities. We use a 0.5 step size in logarithm of the total column density.

In each panel (except for $\mathrm{b}$, where $\Gamma=1.5$ and $\xi=10^{4}$ ) we see the same trend in the structure while increasing the value of the total column density. For the lowest total column densities the temperature does not decrease strongly, because the cloud is thin enough to exist only in the high temperature equilibrium. In such a case the warm absorber is well modeled by a constant density slab with low density of the order of $n=10^{11} \mathrm{~cm}^{-3}$. A temperature drop appears when $N_{\mathrm{H}_{\mathrm{tot}}}$ increases, and the highly

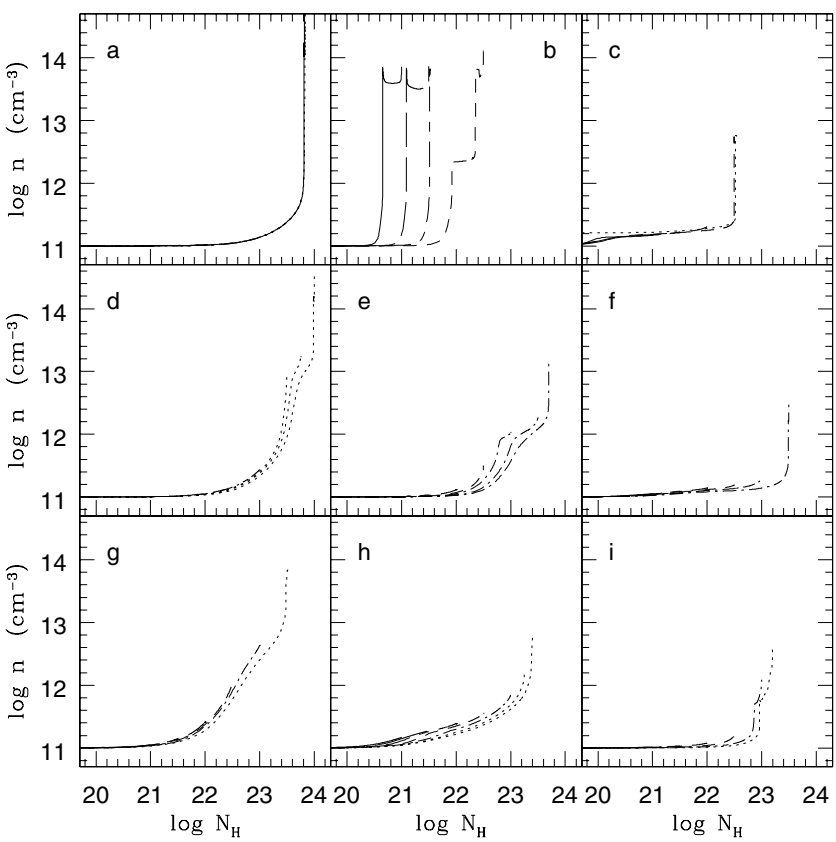

Fig. 4. The density profiles within the irradiated clouds at constant pressure. The different panels show the dependence on the total column density, starting with $\log N_{\mathrm{H}_{\mathrm{tot}}}=21$, with step sizes of 0.5 . The step size decreases to 0.1 when approaching the value of $\log N_{\mathrm{H}_{\mathrm{tot}}}^{\mathrm{Max}}$. Panels a), b), c) show the results for $\xi=10^{5}, 10^{4}$ and $10^{3}$, and for $\Gamma=1.5$. Panels d), e), f) show respectively the same ionization parameters but for $\Gamma=2$. The last three panels, $\mathbf{g}), \mathbf{h})$, i), present the cases for $\Gamma=2.5$.

ionized hot layer is complemented by geometrically thin, dense zones even reaching $n=5 \times 10^{14} \mathrm{~cm}^{-3}$. Since the transition to solutions with the outer cool layer occurs rapidly, we had to decrease the step in $\log N_{\mathrm{H}_{\text {tot }}}$ to 0.1 , for the last two curves in almost each panel in order to correctly resolve the structure.

The exceptional case of $\Gamma=1.5, \xi=10^{4}$ (panel b in Fig. 3) which was discussed at the end of Sect. 3 shows a strong temperature jump even for $N_{\mathrm{H}_{\mathrm{tot}}}=10^{21} \mathrm{~cm}^{-2}$. The dense layer on the back of the illuminated face has an unphysical temperature bump. Such a layer has a non negligible optical thickness and its modeled spectrum possesses strong absorption (see section below).

In Fig. 5 we present thermal equilibrium curves, $T(\Xi)$, for several clouds. The value of the dynamical ionization parameter across the cloud was computed using relation $\Xi=P_{\text {rad }} / P_{\text {gas }}$. From this figure we see the difference between our models and those when two constant density slabs are used to fit a data. We propose the warm absorber as a single cloud that passes through all ionization states, and that each layer interacts radiatively with others as usually done in classical atmospheric calculations.

For one specific case $\left(\Gamma=2.0, \xi=10^{4}\right)$ we calculate models with the maximum value of $N_{\mathrm{Htot}}^{\mathrm{Max}}$ assuming two values of the turbulent velocity: $100 \mathrm{~km} \mathrm{~s}^{-1}$ and $300 \mathrm{~km} \mathrm{~s}^{-1}$. The turbulence affects the thermal structure of the cloud (see Fig. 6). The interior of the turbulent cloud is hotter than the interior of a "static" cloud, and the cloud interior is less dense.

\section{Modeled spectra}

The transmitted spectra for the whole set of models are presented in Figs. 7-9 for $\Gamma=1.5,2$ and 2.5 respectively. All figures are given in the range of energies between 0.1 and $4 \mathrm{keV}$, where most 


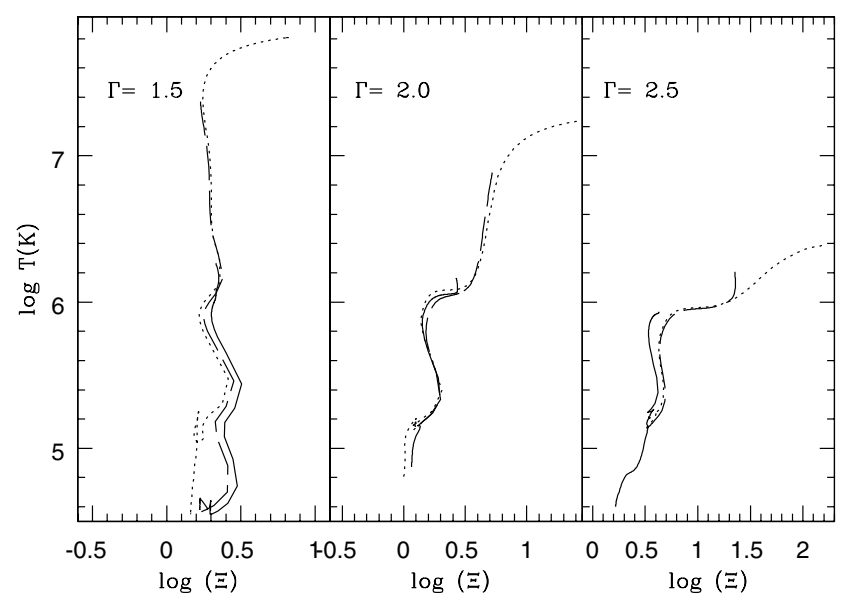

Fig. 5. The thermal equilibrium curves for the clouds at constant pressure. Clouds with $\Gamma=1.5$ are presented in the left panel, with $\Gamma=2.0$ in the middle panel, and $\Gamma=2.5$ in the right panel. Dotted line describes clouds with $\xi=10^{5}$, dashed line $-\xi=10^{4}$, and solid line $-\xi=10^{2}$. In all cases we show clouds with maximum total column density.

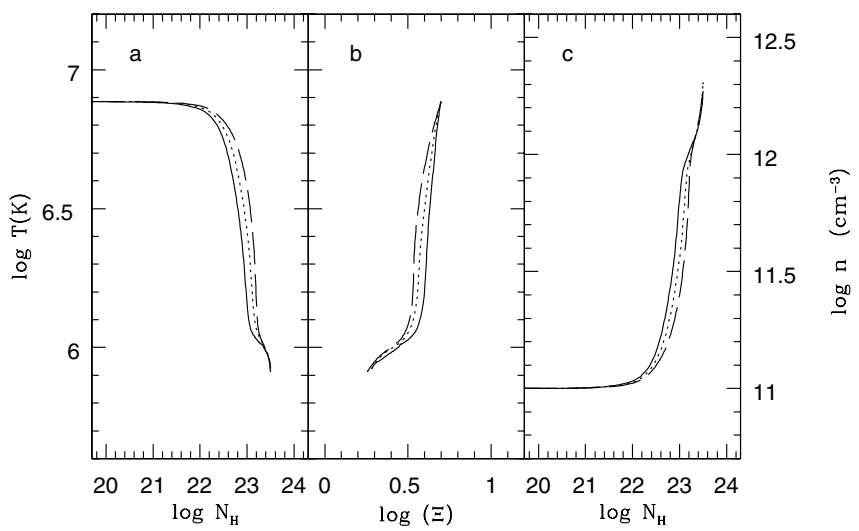

Fig. 6. The structure of the warm absorber in the case of turbulent velocity for $\Gamma=2.0, \xi=10^{4}$ and maximum column density $N_{\mathrm{Htot}}^{\mathrm{Max}}$. Panel a) shows the temperature structure, b) thermal equilibrium curve $T(\Xi)$ and panel c) the density structure. The solid line represents the case with $v_{\text {turb }}=0$, dotted line with $v_{\text {turb }}=100 \mathrm{~km} \mathrm{~s}^{-1}$, and dashed line with $v_{\text {turb }}=300 \mathrm{~km} \mathrm{~s}^{-1}$.

lines are present. The energy ranges of iron lines from $6 \mathrm{keV}$ to $8 \mathrm{keV}$ are presented and discussed in Sect. 6.

For high ionization parameters and low total column densities the spectra are featureless since the matter is almost fully ionized and only the changes in the continuum are caused by Comptonization. This effect is clearly seen in the upper panels of Figs. 7 and 8, where the whole outgoing continuum is lowered. The Compton heating reaches almost $50 \%$ of the total heating, therefore the determination of the exact value of Compton heating was very important in such cases and we performed it iteratively using the Monte Carlo code NOAR.

In the spectra corresponding to higher total column densities we observe many absorption features. The spectrum in the energy range from $0.8 \mathrm{keV}$ up to $2 \mathrm{keV}$ is especially strongly absorbed due to many transitions of highly ionized heavy elements.

The comparison of spectra for the same total column density of the cloud but for different ionization parameters is shown in Fig. 10. The upper panel presents the results for slabs with $N_{\mathrm{H}_{\mathrm{tot}}}=10^{22} \mathrm{~cm}^{-2}$ illuminated by radiation of photon index $\Gamma=1.5$. The different spectra from the top to the bottom represent the cases for $\xi=10^{5}, 10^{4}$ and $10^{3}$ respectively. The same

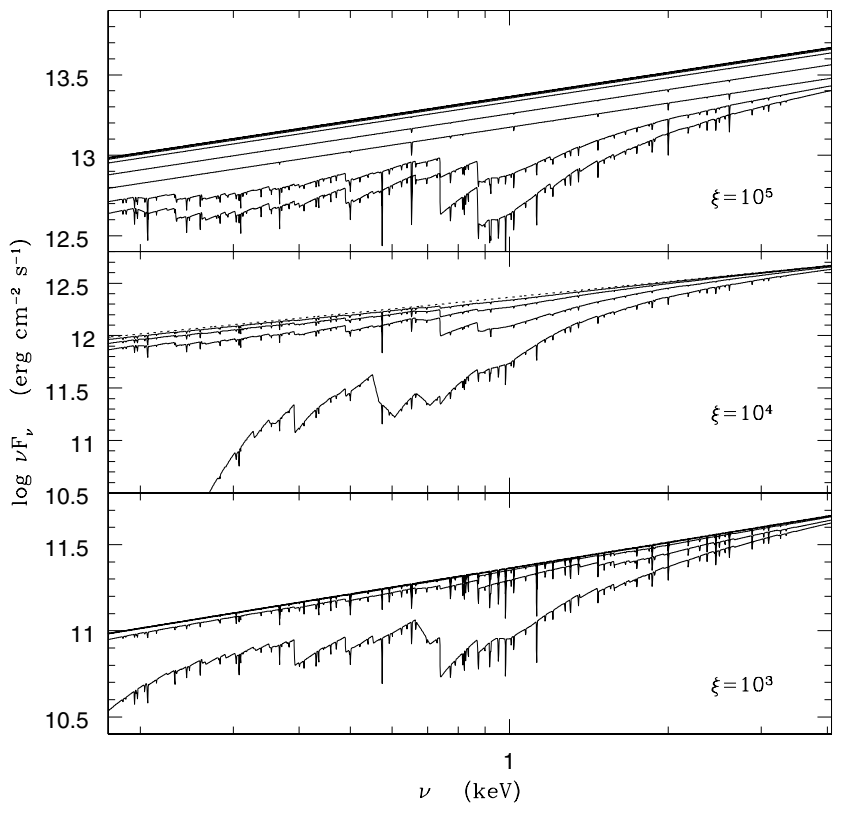

Fig. 7. Set of transmission spectra for the photon index of the incident continuum $\Gamma=1.5$ and $\xi=10^{5}$ (upper panel), $\xi=10^{4}$ (middle panel) and $\xi=10^{3}$ (lower panel). The results for a range of $\log N_{\mathrm{H}_{\mathrm{tot}}}$ are shown in each panel starting from $\log N_{\mathrm{H}}=21$. to $\log N_{\mathrm{H}_{\mathrm{tot}}}^{\mathrm{Max}}$.

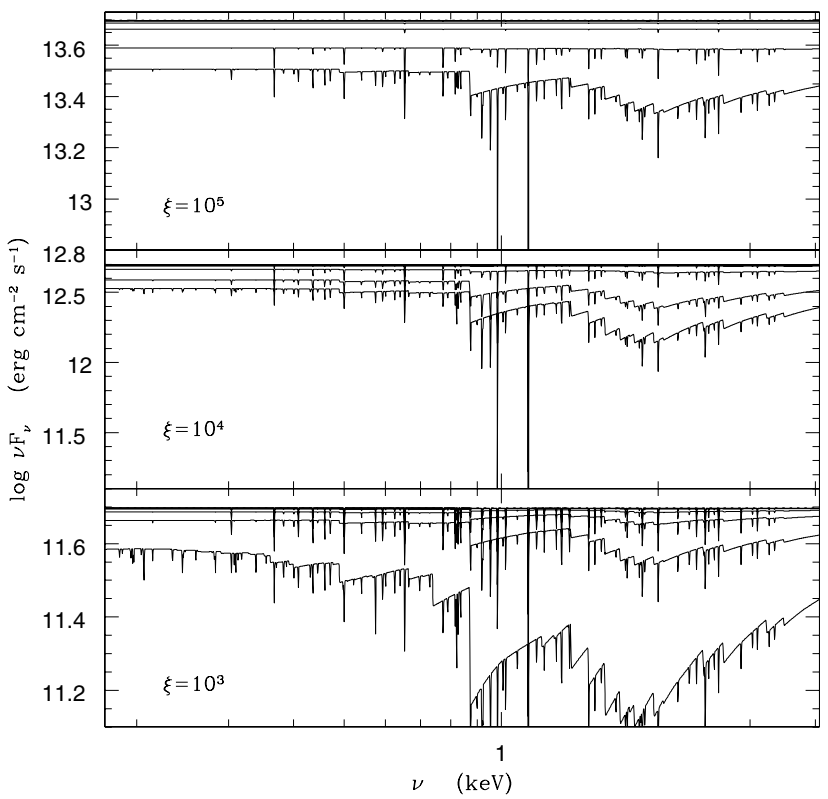

Fig. 8. Set of transmission spectra for the photon index of the incident continuum $\Gamma=2.0$ and $\xi=10^{5}$ (upper panel), $\xi=10^{4}$ (middle panel) and $\xi=10^{3}$ (lower panel). The results for a range of $\log N_{\mathrm{H}_{\mathrm{tot}}}$ are shown in each panel starting from $\log N_{\mathrm{H}}=21$. to $\log N_{\mathrm{H}_{\mathrm{tot}}}^{\mathrm{Max}}$.

sequence of ionization parameters is presented in the middle panel for $N_{\mathrm{H}_{\mathrm{tot}}}=10^{23} \mathrm{~cm}^{-2}$ and $\Gamma=2.0$, and in the lower panel for $N_{\mathrm{H}_{\mathrm{tot}}}=3.16 \times 10^{22} \mathrm{~cm}^{-2}$ and $\Gamma=2.5$.

The spectrum in the case of $\Gamma=1.5$ and $\xi=10^{4}$ is again exceptional because of the numerical problems mentioned above. In this spectrum many absorption edges are seen. This is because the optically thick layer on the back of the illuminated cloud is also geometrically thick and the absorption is much stronger. Additionally, the highly ionized optically thin layer to the front of the illuminated cloud is thin enough to not interact 


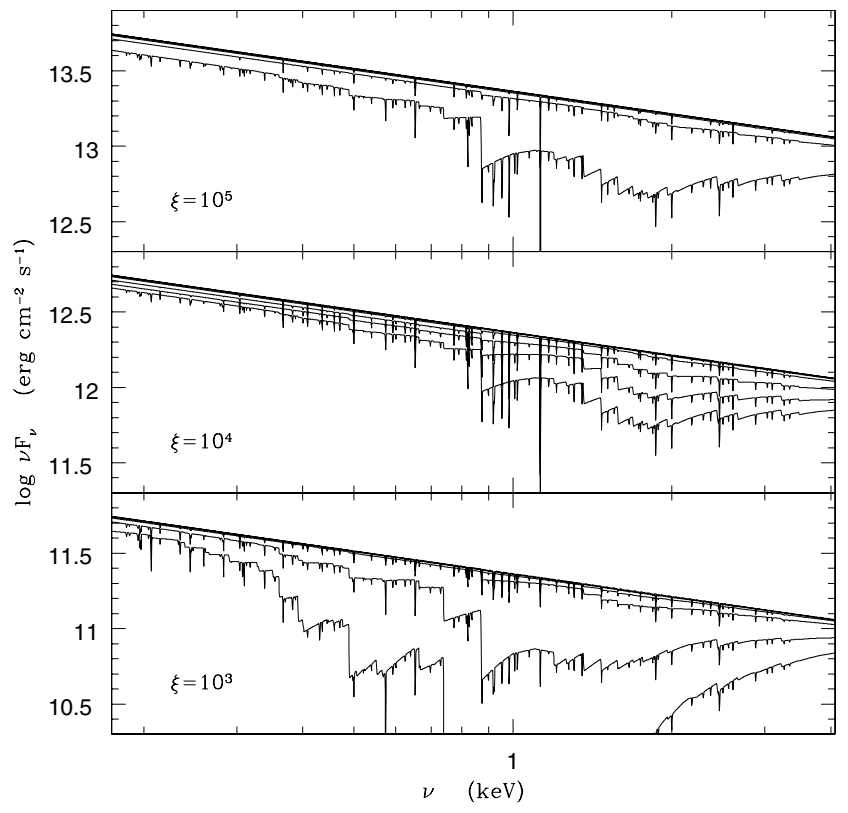

Fig. 9. Set of transmission spectra for the photon index of the incident continuum $\Gamma=2.5$ and $\xi=10^{5}$ (upper panel), $\xi=10^{4}$ (middle panel) and $\xi=10^{3}$ (lower panel). The results for a range of $\log N_{\mathrm{H}_{\mathrm{tot}}}$ are shown in each panel starting from $\log N_{\mathrm{H}}=21$ to $\log N_{\mathrm{H}_{\mathrm{tot}}}^{\mathrm{Max}}$.

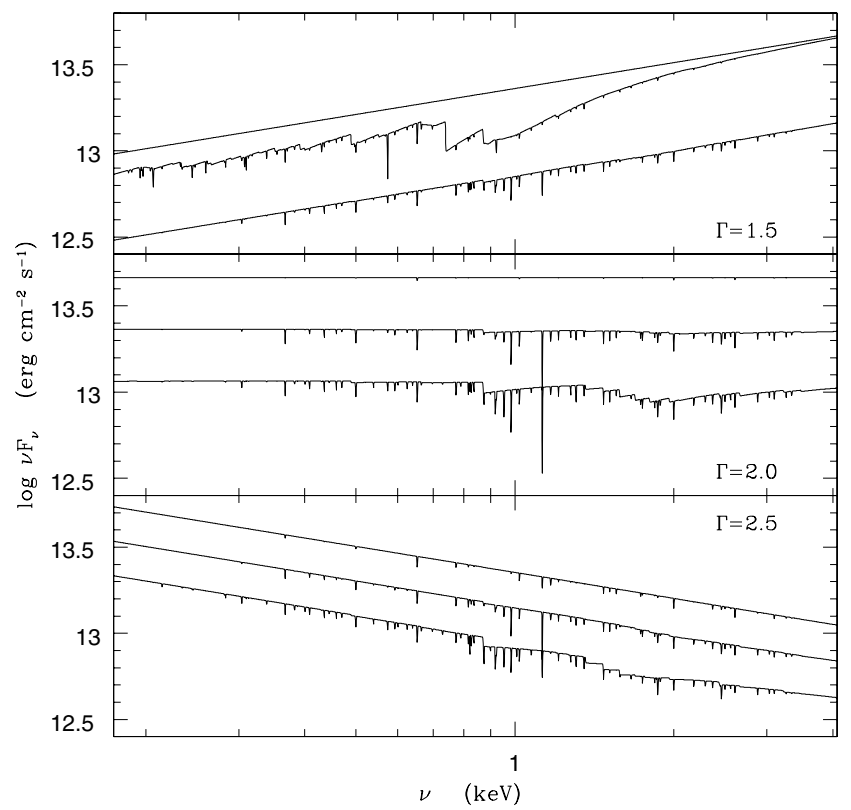

Fig. 10. Comparison of spectra for the same $N_{\mathrm{H}_{\mathrm{tot}}}$, but for different ionization parameters. In the upper panel we compare clouds of $N_{\mathrm{H}_{\mathrm{tot}}}=$ $10^{22} \mathrm{~cm}^{-2}$ illuminated by radiation with photon index $\Gamma=1.5$, middle panel $N_{\mathrm{H}_{\mathrm{tot}}}=10^{23} \mathrm{~cm}^{-2}$ and $\Gamma=2.0$, and lower panel $N_{\mathrm{H}_{\mathrm{tot}}}=3.16 \times$ $10^{22}$ and $\Gamma=2.5$. In each panel lines from the top to the bottom present cases for $\xi=10^{5}, 10^{4}$ and $10^{3}$ respectively.

with X-ray radiation, explaining the lack of lines above $1 \mathrm{keV}$ in this spectrum.

Other spectra show an increase in the number of lines with decreasing ionization parameter. Also less energetic lines (below $1 \mathrm{keV}$ ) are much more numerous for lower $\xi$. This is because the back side of an illuminated cloud reaches lower temperatures and higher densities, and also lower ionization states for lower $\xi$.

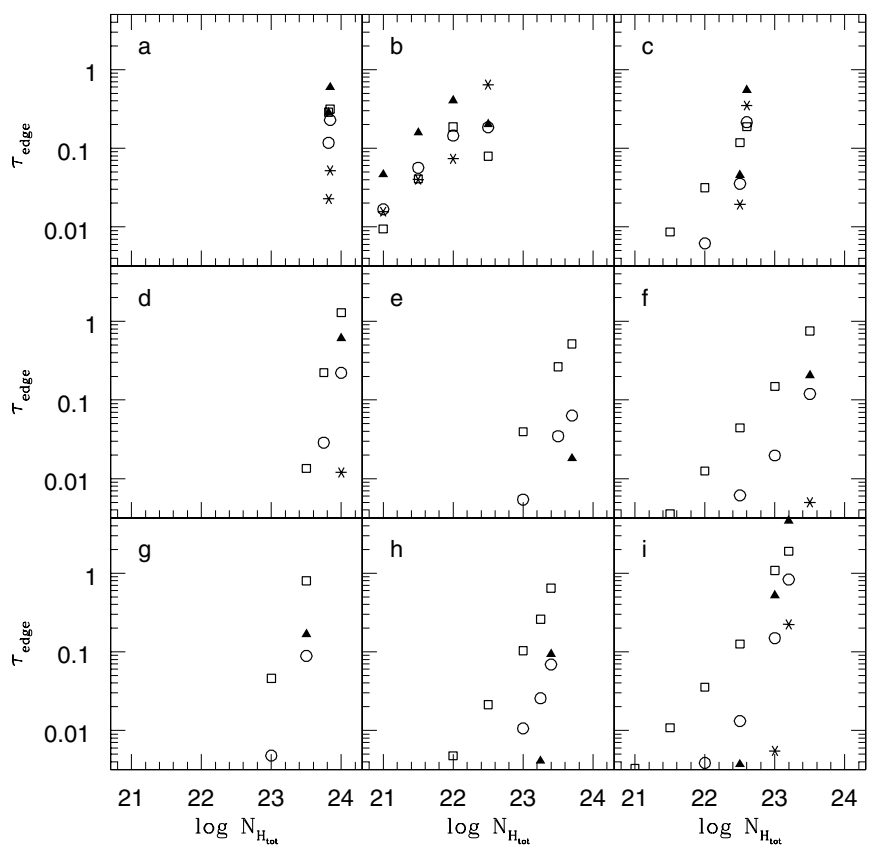

Fig. 11. The strength of absorption edges for irradiated clouds at constant pressure. The different panels show the dependence on the total column density, starting with $\log N_{\mathrm{H}_{\text {tot }}}=21$, with step size of 0.5 . The step size decreases to 0.1 when approaching the value $\log N_{\mathrm{H}_{\mathrm{tot}}}^{\mathrm{Max}}$. Panels a), b), c) show the results for $\xi=10^{5}, 10^{4}$ and $10^{3}$, and for $\Gamma=1.5$. Panels d), e), f) show respectively the same ionization parameters but for $\Gamma=2$. Last three panels: $\mathbf{g}$ ), h), i) present the cases for $\Gamma=2.5$. Filled triangles denote OVII edges, open squares are OVIII, stars are $\mathrm{CV}$, and open circles are $\mathrm{CVI}$ edges.

\subsection{Absorption edges}

The detection of absorption edges in the spectra of AGN puts direct constraints on the total column density of the warm absorber. Therefore, we show in Fig. 11 the strength of the main ionization edges in our synthetic spectra for comparison with observations. Models with $\Gamma=1.5$ are presented in panels a, b, c for $\xi=10^{5}, 10^{4}$ and $10^{3}$ respectively. The same sequence of $\xi$, but for $\Gamma=2$ is shown in panels $\mathrm{d}$, e, $\mathrm{f}$, and the softest intrinsic spectrum with photon index 2.5 is presented in panels $g, h$ and $i$.

The strength of an edge is measured by its total optical thickness defined by the total absorption plus scattering opacity coefficient multiplied by the density and integrated over the geometrical size of the cloud. We show only OVII (filled triangles), OVIII (open squares), CV (stars), and CVI (open circles) edges above $\tau_{\text {edge }}=0.003$, which can be resolved using current X-ray satellites.

Again panel $b$ shows the exceptional case, and we see that the ionization edges are strong for the reason mentioned in the previous section. In the other cases the edges become visible only when the total column densities are higher than $10^{23} \mathrm{~cm}^{-2}$ for $\xi=10^{5}$ (panels a, d, g). This minimum total column density decreases with decreasing ionization parameter reaching $10^{22} \mathrm{~cm}^{-2}$ for $\xi=10^{3}$.

\subsection{Strength of the absorption lines}

Several physical conditions influence the amount of energy absorbed in lines: the heavy element abundance, temperature of the medium, the hardness of the illuminating continuum, the column densities of particular ions, and the turbulent velocity of the 


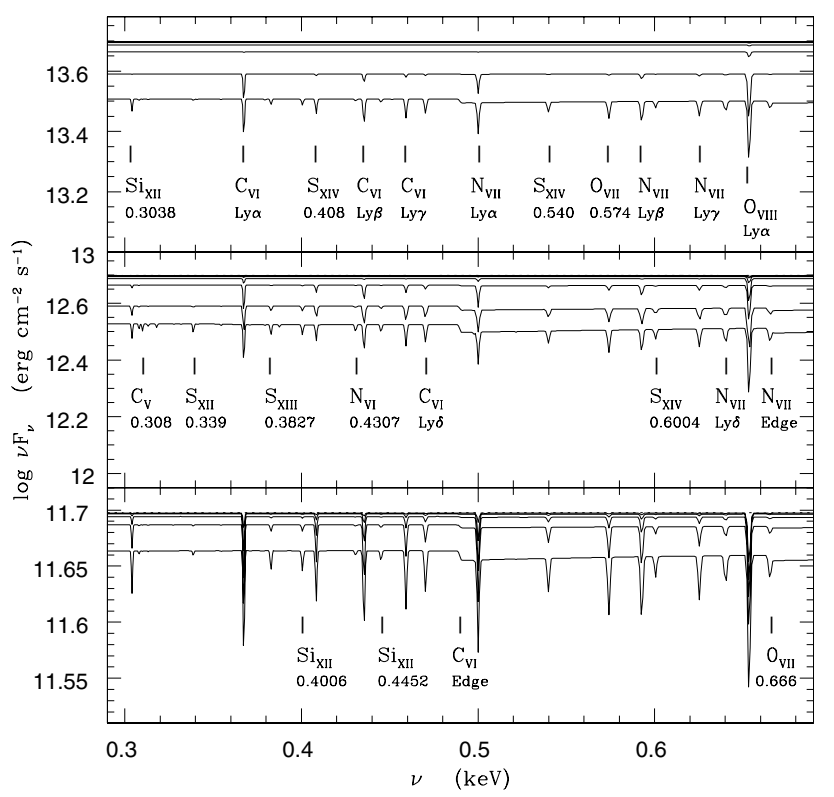

Fig. 12. The expanded version of Fig. $8(\Gamma=2)$ in the softest energy range between 0.3 and $0.7 \mathrm{keV}$. Models for different $\xi=10^{5}, 10^{4}$, and $10^{3}$ are presented in upper, middle and lower panel respectively. The lowest continuous lines in each panel correspond to $N_{\mathrm{H}_{\mathrm{tot}}}^{\mathrm{Max}}$ appropriate for the set of $\Gamma$ and $\xi$ as seen in Fig. 2.

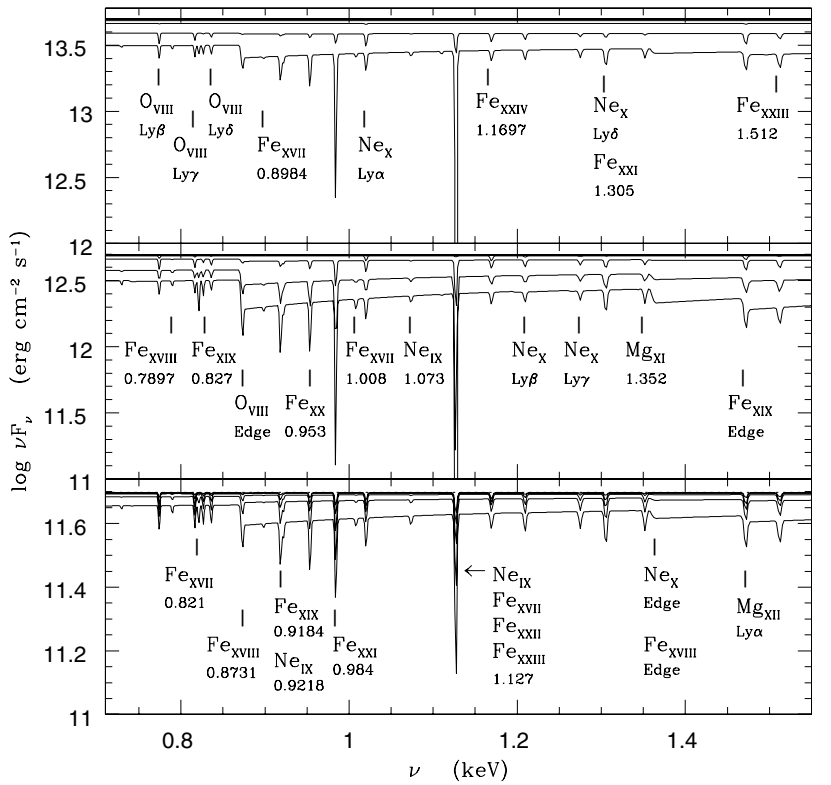

Fig. 13. The same as Fig. 12 but for energy range between 0.7 and $1.5 \mathrm{keV}$.

absorbing matter. It is not obvious which condition is the most important, but our models consider variations in the illuminating spectrum, the total column density, the ionization parameter, and the turbulent velocity (in a limited way).

In order to study the strength of absorption lines in our spectra, in Figs. 12-15 we show expanded views of the spectra corresponding to $\Gamma=2$ (see Fig. 8). The range between 0.3 and $0.7 \mathrm{keV}$ is shown in Fig. 12, between 0.7 and $1.5 \mathrm{keV}$ in Fig. 13, from 1.5 to $3.5 \mathrm{keV}$ is presented in Fig. 14, and from 6.2 to $9 \mathrm{keV}$ is shown in Fig. 15.

All lines in models with zero turbulent velocities are weak (see Table 1), with equivalent widths smaller than $1 \mathrm{eV}$, and only in a few cases (SiXIV Ly $\alpha$, SXVI Ly $\alpha$, FeXXVI Ly $\alpha$ up to

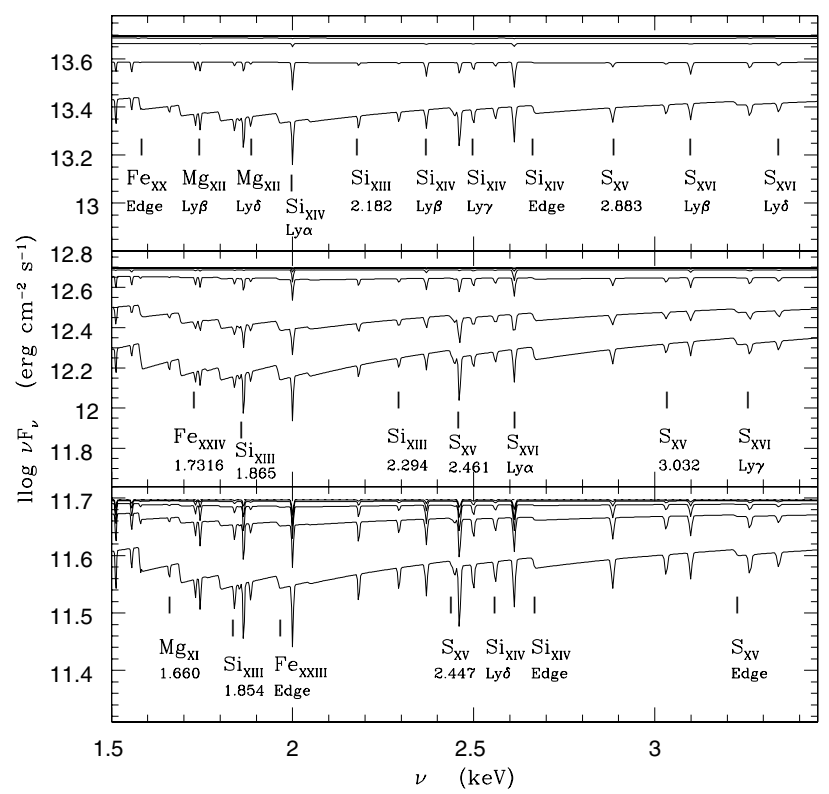

Fig. 14. The same as Fig. 12 but for energy range between 1.5 and $3.5 \mathrm{keV}$.

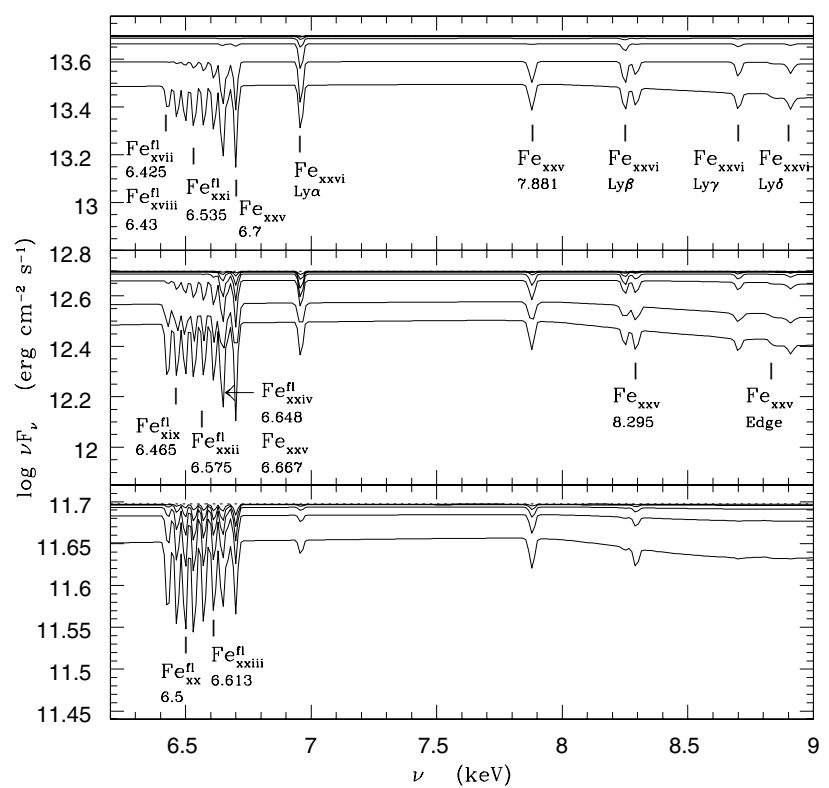

Fig. 15. The same as Fig. 12 but for energy range between 6.2 and $9 \mathrm{keV}$.

Ly $\gamma$, also some fluorescent iron lines) they do reach a few eVs. For a decreasing ionization parameter $\xi$ (going from the top to the bottom panels on each figure) we see that the lines become stronger and are present even for lower total column densities.

Some lines do not change very much with increasing column density. These lines are predominantly from the most highly ionized species. Lines from ions of slightly lower ionization degree are not present in cases when the total column density is low, because the dense zone does not exist at the back of the illuminated cloud.

The influence of turbulent motion on the strength of absorption lines is presented in Fig. 16. The continuum is slightly modified in the $2-3 \mathrm{keV}$ range but absorption lines are naturally much more intense (see Fig. 16).

Quantitative results for turbulent line intensities are shown in Table 1, Cols. 6 and 7. The equivalent widths of those lines 
Table 1. Equivalent widths in $[\mathrm{eV}]$ of absorption lines from clouds with different ionization parameters at the surface.

\begin{tabular}{|c|c|c|c|c|c|c|c|c|}
\hline$\Gamma$ & & 2.0 & & 2.5 & 2.0 & 2.0 & Observed & Ref. \\
\hline$\xi$ & $10^{5}$ & $10^{4}$ & $10^{3}$ & $10^{3}$ & $10^{4}$ & $10^{4}$ & & \\
\hline $\log \left(N_{\mathrm{H}_{\mathrm{tot} 1}}\right)$ & 23.5 & 23.5 & 23.5 & 23.2 & 23.5 & 23.5 & & \\
\hline & & & & & $v_{\text {turb }}=100 \mathrm{~km} \mathrm{~s}^{-1}$ & $v_{\text {turb }}=300 \mathrm{~km} \mathrm{~s}^{-1}$ & & \\
\hline CVI Ly $\alpha$ & 0.18 & 0.24 & 0.25 & 0.31 & 0.5 & 1.27 & $0.98 \pm 0.33$ & (2) \\
\hline NVII Ly $\alpha$ & 0.19 & 0.28 & 0.31 & 0.4 & 0.63 & 1.55 & $1.33 \pm 0.24$ & (2) \\
\hline OvIII Ly $\alpha$ & 0.54 & 0.65 & 0.79 & 0.98 & 1.13 & 2.97 & $1.85 \pm 0.55$ & (1) \\
\hline OVIII (0.7743) & 0.38 & 0.44 & 0.44 & 0.38 & 1.05 & 2.76 & $1.77 \pm 0.44$ & (1) \\
\hline FeXVII (0.8211) & 0.002 & 0.35 & 0.93 & 0.90 & 0.7 & 1.24 & $1.42 \pm 0.28$ & (1) \\
\hline FeXIX (0.8266) & 0.002 & 0.33 & 0.52 & 0.47 & 0.97 & 2.50 & $0.83 \pm 0.24$ & (1) \\
\hline FeXVIII (0.8731) & 0.01 & 0.45 & 0.88 & 0.47 & 1.0 & 2.37 & $1.52 \pm 0.34$ & (1) \\
\hline FeXVII (0.8984) & - & 0.1 & 0.23 & 0.29 & 0.2 & 0.2 & $1.43 \pm 0.52$ & (2) \\
\hline FeXIX (0.9184) & 0.1 & 0.96 & 1.17 & 0.96 & 1.47 & 3.48 & $1.84 \pm 0.54$ blend & (2) \\
\hline NeIX (0.9218) & 0.03 & 0.33 & 0.63 & 1.25 & 0.8 & 1.48 & $4.13 \pm 0.48$ & (1) \\
\hline & & & & & & & $2.6 \pm 1.48$ & (2) \\
\hline & & & & & & & $1.33_{-0.30}^{+0}$ & (3) \\
\hline & & & & & & & $1.09_{-0.34}^{+0.35}$ & (4) \\
\hline FeXX (0.953) & 0.2 & 1.22 & 1.22 & 0.97 & 1.69 & 3.95 & $4.88 \pm 0.42$ blend & (1) \\
\hline $\operatorname{Nex} \operatorname{Ly} \alpha$ & 0.6 & 0.77 & 0.86 & 0.78 & 1.54 & 4.08 & $3.20 \pm 0.24$ blend & (1) \\
\hline NeIX (1.127) & 0.003 & 0.13 & 0.27 & 0.27 & 0.2 & 0.2 & all complex & \\
\hline FeXVII (1.127) & - & 0.04 & 0.2 & 0.22 & 0.07 & 0.07 & $4.66 \pm 0.33$ & (1) \\
\hline FeXXIII (1.127) & 0.964 & 3.82 & 2.86 & 1.12 & 4.54 & 9.55 & $2.15 \pm 0.82$ & (2) \\
\hline NeX (1.209) & 0.36 & 0.54 & 0.55 & 0.46 & 1.40 & 3.42 & $2.3 \pm 0.24$ & (1) \\
\hline $\operatorname{MgXII} \operatorname{Ly} \alpha$ & 0.74 & 0.99 & 1.08 & 0.82 & 2.09 & 5.45 & $4.39 \pm 0.24$ & (1) \\
\hline FeXXIV (1.556) & 0.43 & 0.5 & 0.44 & 0.1 & 1.69 & 3.94 & $1.84 \pm 0.23$ & (1) \\
\hline MgXII (1.745) & 0.4 & 0.67 & 0.7 & 0.56 & 1.83 & 4.09 & $1.75 \pm 0.22$ & (1) \\
\hline SiXIII (1.865) & 0.46 & 1.47 & 1.92 & 1.91 & 2.66 & 6.6 & $4.18 \pm 0.20$ & (1) \\
\hline & & & & & & & $3.37 \pm 1.68$ & (2) \\
\hline & & & & & & & $1.49_{-0.73}^{+0.76}$ & (4) \\
\hline SixIV Ly $\alpha$ & 1.26 & 1.78 & 1.80 & 1.13 & 3.12 & 7.99 & $6.61 \pm 0.26$ & (1) \\
\hline & & & & & & & $3.87 \pm 1.94$ & (2) \\
\hline & & & & & & & $4.19_{-1.29}^{+1.42}$ & (4) \\
\hline SiXIV (2.370) & 0.8 & 0.97 & 0.94 & 0.71 & 2.72 & 6.64 & $5.03 \pm 0.72$ blend & (1) \\
\hline SXV (2.461) & 0.72 & 2.23 & 2.56 & 2.02 & 3.62 & 8.73 & $4.49 \pm 0.59$ & (1) \\
\hline SiXIV (2.500) & 0.48 & 0.84 & 0.85 & 0.63 & 2.33 & 4.48 & $1.96 \pm 0.71$ & (1) \\
\hline $\operatorname{SxVI} \operatorname{Ly} \alpha(2.611)$ & 1.51 & 1.93 & 1.81 & 0.96 & 3.68 & 9.3 & $5.88 \pm 0.66$ & (1) \\
\hline & & & & & & & $3.85_{-2.80}^{+2.86}$ & (4) \\
\hline SXV (2.883) & 0.3 & 0.93 & 1.04 & 0.86 & 2.87 & 6.15 & $1.81 \pm 0.67$ & (1) \\
\hline $\operatorname{Sxv}(3.032)$ & 0.13 & & 0.9 & 0.72 & 2.19 & 3.31 & $2.82 \pm 0.82$ & (1) \\
\hline SXVI (3.100) & 0.9 & 1.11 & 1.04 & 0.69 & 3.10 & 6.48 & $2.87 \pm 0.78$ blend & (1) \\
\hline FexXV (6.700) & 6.74 & 9.0 & 5.35 & 0.25 & 11.99 & 25.19 & $13.76 \pm 5.07$ & (1) \\
\hline FexXV (7.881) & 3.37 & 3.57 & 2.22 & 0.047 & 8.25 & 17.75 & $33.06 \pm 19.04$ & (1) \\
\hline
\end{tabular}

(1) NGC 3783, 900 ks Chandra Kaspi et al. (2002); (2) NGC 5548, 86.4 ks Chandra Kaastra et al. (2002); (3) Mrk 509, 59 ks Chandra Yaqoob et al. (2003); (4) NGC 4051, 81.5 ks Chandra Collinge et al. (2001).

increase by a factor of a few. The same trend was seen in constant pressure models of Różańska et al. (2004). A higher value of the turbulent velocity leads to overprediction of the line strength. However, some predicted iron lines are still definitively weaker than in the data.

We will consider more systematically the effects of turbulent velocities in future work. Metal abundance is another free parameter in our model that should be studied more carefully in the forthcoming paper.

\section{Influence of the warm absorber on the relativistic iron line}

A broad, relativistically smeared iron line was discovered in a number of sources, MCG-6-30-15 being the first discovered and the best example of this phenomenon (Tanaka et al. 1995).
It is produced by the reflection of the hard X-ray continuum on the disk surface very close to the black hole. If the radiation originating at the disk passes through the warm absorber before it reaches the observer, the spectral shape of the line is modified.

To show this effect, we considered an example of the broad disk line. We have assumed that the disk is illuminated by a random distribution of flares following Czerny et al. (2004). Each flare produces a hot spot below, and each spot emits locally like an illuminated disk atmosphere. The final emissivity of the disk was computed by integrating over all disk radii. The black hole was assumed to be rotating, with a Kerr parameter $a=0.95$ and an inclination to the observer of $i=30^{\circ}$. All effects of general relativity were included.

The spectral shape of the reflected disk component from this model (without any effect of the warm absorber) is shown in Fig. 17 as the dashed line in both panels a and b. Since the local reflection comes from the partially ionized disk surface, 

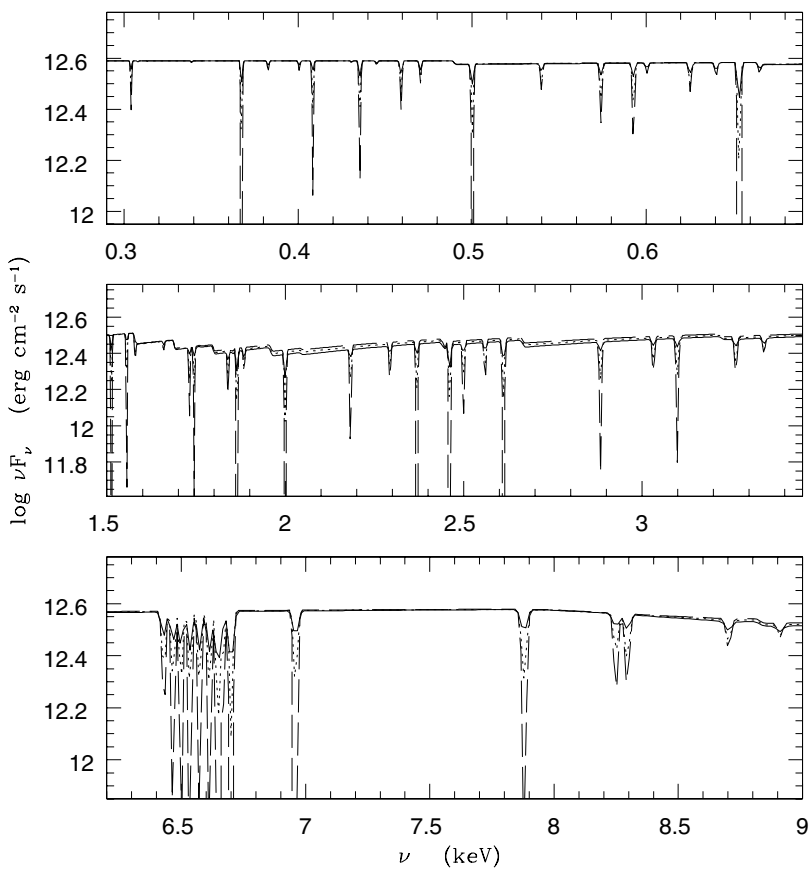

Fig. 16. Set of transmission spectra in the case of turbulent velocity for $\Gamma=2.0, \xi=10^{4}$ and a maximum column density $N_{\mathrm{H}_{\mathrm{tot}}}^{\mathrm{Max}}$. The solid line represents the case with $v_{\text {turb }}=0$, dotted line with $v_{\text {turb }}=100 \mathrm{~km} \mathrm{~s}^{-1}$, and dashed line with $v_{\text {turb }}=300 \mathrm{~km} \mathrm{~s}^{-1}$.

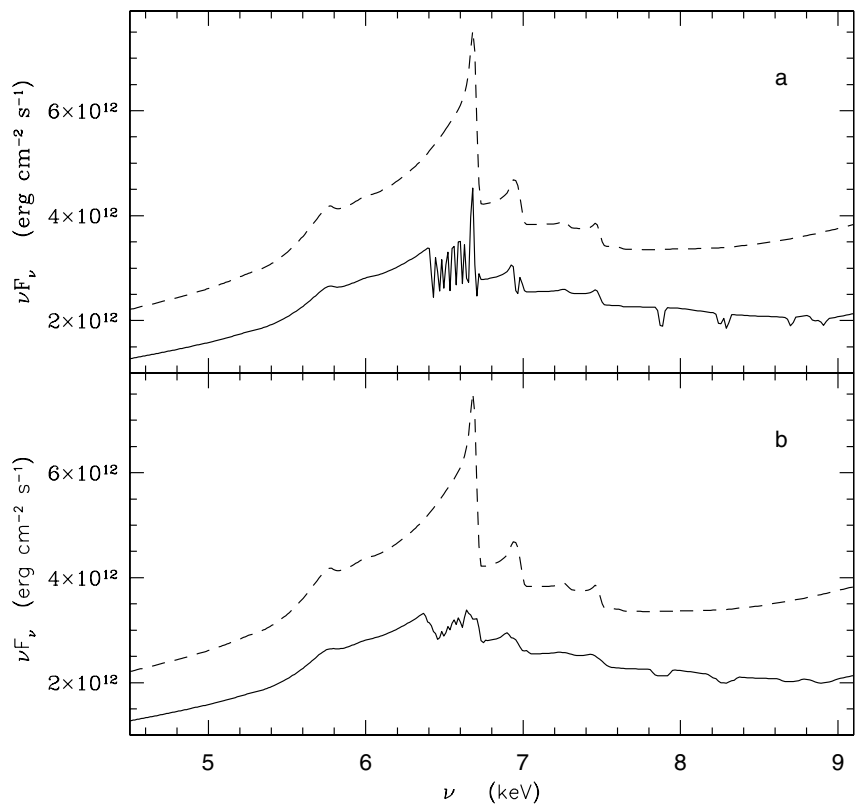

Fig. 17. An example of the effect of absorption on the shape of a relativistically smeared disk line. The unabsorbed disk spectrum is marked by a dashed line, while the solid line represents the spectrum with two resolutions: a resolution of 390 in panel a), and the resolution of Chandra HEG at $6 \mathrm{keV}$ i.e. 200 in panel b).

several components of the iron line complex are still visible in the resulting spectrum, although they are relativistically smeared. However, if we see the nucleus through the warm absorber with a total column density $N_{\mathrm{H}_{\mathrm{tot}}}=4.5 \times 10^{23}$ and an ionization parameter $\xi=10^{4}$ (second spectrum from the bottom in Fig. 15 in the middle panel), the overall spectrum is suppressed and the narrow absorption features appear superimposed on the broad emission line profile.
In panel a of Fig. 17 we show the result (solid line) calculated with the resolution used in our computations, $E / \Delta E=390$, while in panel $\mathrm{b}$ we show the same result degraded to the resolution of $E / \Delta E=200$ characteristic of the Chandra HEG instrument at $6 \mathrm{keV}$.

We see that a strong absorption modifies the overall shape of the broad iron line. The absorbed shape of line seems to be more symmetric and closer to a Gaussian shape. Also the fitted representative energy (mean energy of the line) would be lower than derived for the intrinsic spectrum. The situation is therefore similar to the case of UV spectra where we see narrow absorption features in BLR lines.

\section{Comparison with observations}

Recent observations of Sy1 or Sy1.5 galaxies show the presence of absorption lines in soft X-ray spectra from Chandra or $X M M$ satellites. The number of detected lines depends on the resolution and sensitivity of the detector, and the best observations were obtained for NGC 3783 (Kaspi et al. 2002; Behar et al. 2003; Netzer et al. 2003; Krongold et al. 2003). A 900 ks observation allowed for the detection of over a few tens of lines. In the cases of about $90 \mathrm{ks}$ observations or shorter, only a few lines were detected and fitted, as in the case of NGC 5548, Mrk 509, TonS180, PG 1211+143, or NGC 7469 (Blustin et al. 2003). Therefore, it is hard to fit our models to those observations, but we make some comparison by examining parameters already fitted by other authors.

The data suggest that the total column density of the warm absorber is never higher than $\sim 10^{23} \mathrm{~cm}^{-3}$ (Piconcelli et al. 2004). In our models we have a physical limit to the column density because of the presence of thermal instabilities. For each spectral index and ionization parameter there is a maximum value of the column density for which a warm absorber exists (see Fig. 2). In all cases the values of this maximum total column density agree with the column densities determined from observations of different objects.

In a stratified medium it is difficult to determine the plasma parameters by directly comparing the $E W \mathrm{~s}$ of individual lines. Therefore, in Table 2 we present the ratios of the $E W$ s of lines for the same ion. In the first column names of ratios are given, in the second column those ratios are computed theoretically assuming that lines are optically thin (see formula 2 in Różańska et al. 2004). We have listed only those line ratios detected by $\mathrm{X}$-ray satellites. Columns from 3 to 7 present the observed ratios assuming the most pessimistic error, when the numerator has its maximal value, and the denominator has its minimal value (see Table 2).

The observed line ratios are usually much lower than predicted in the optically thin medium limit. Therefore, observations strongly suggest that lines are optically thick and radiative transfer is required to predict equivalent widths.

The same ratios computed for our models are presented in Table 3. For each set of models with spectral index and ionization parameter, the ratios are of the order of those observed when we consider clouds with higher total column densities. We conclude here that when the warm absorber has a total column density higher than $10^{22} \mathrm{~cm}^{-2}$ the resonance lines of the main ions become optically thick and radiative transfer should be used to model them. For instance, the most popular $\mathrm{Ly}_{\alpha}$ lines of OVIII, NeX, MgXII, SixIV, and SXVI for $\xi=10^{4} \Gamma=2.0$ and $\log N_{\mathrm{H}_{\mathrm{tot}}}=$ 23.5 have optical depths integrated over the whole cloud equal respectively $977,363,234,436$, and 216 . 
Table 2. Ratios of Equivalent Widths of lines from the same ion in the optically thin approximation. For comparison the results from observations are given if available. We calculate the error in the most pessimistic way, when the numerator has its maximal value $E W+\Delta E W$, and the denominator has its minimal value $E W-\Delta E W$.

\begin{tabular}{|c|c|c|c|c|c|c|}
\hline Name of ratio & $\begin{array}{l}\text { Theory } \\
f_{i j} / f_{i k}\end{array}$ & $\begin{array}{l}\text { NGC 3783 } \\
\text { Kaspi et al. } \\
\text { (2002) }\end{array}$ & $\begin{array}{l}\text { NGC 5548 } \\
\text { Kaastra et al. } \\
(2002)\end{array}$ & $\begin{array}{l}\text { Mrk 509 } \\
\text { Yaqoob et al. } \\
(2003)\end{array}$ & $\begin{array}{l}\text { NGC 4051 } \\
\text { Collinge et al. } \\
\text { (2001) }\end{array}$ & $\begin{array}{l}\text { Ton S180 } \\
\text { Różańska et al. } \\
\text { (2004) }\end{array}$ \\
\hline $\mathrm{CV}_{(0.3079) /(0.3545)}$ & 4.6 & - & $3.85 \pm 23.15$ & - & - & - \\
\hline $\mathrm{CVI}_{(\operatorname{Ly} \alpha) /(\operatorname{Ly} \beta)}$ & 8.49 & - & $2.33 \pm 2.99$ & - & - & - \\
\hline $\mathrm{CVI}_{(\mathrm{Ly} \alpha) /(\mathrm{Ly} \gamma)}$ & 14.34 & - & $3.77 \pm 14.94$ & - & - & - \\
\hline $\mathrm{CVI}_{(\mathrm{Ly} \alpha) /(\mathrm{Ly} \delta)}$ & 29.86 & - & - & - & - & $0.62_{-0.59}^{+3.53}$ \\
\hline $\operatorname{NVII}_{(\operatorname{Ly} \alpha) /(\operatorname{Ly} \beta)}$ & 5.25 & $1.53 \pm 4.39$ & $12.09 \pm 1.18$ & $0.86 \pm 0.98$ & - & - \\
\hline $\operatorname{NVII}_{(\operatorname{Ly} \alpha) /(\operatorname{Ly} \gamma)}$ & 14.34 & $3.5 \pm 8.3$ & - & - & - & - \\
\hline $\operatorname{NVII}_{(\operatorname{Ly} \alpha) /(\operatorname{Ly} \delta)}$ & 29.92 & $1.48 \pm 2.27$ & - & - & - & - \\
\hline $\operatorname{OVII}_{(0.574) /(0.6656)}$ & 4.75 & $0.95 \pm 1.76$ & $1.32 \pm 0.58$ & - & $1.11 \pm 1.45$ & - \\
\hline OVII $_{(0.574) /(0.6977)}$ & 1.26 & $0.73 \pm 1.01$ & $2.13 \pm 2.21$ & - & $1.43 \pm 2.48$ & - \\
\hline OVIIII $_{(\mathrm{Ly} \alpha) /(\mathrm{Ly} \beta)}$ & 5.26 & $1.04 \pm 0.75$ & $1.16 \pm 0.55$ & - & $2.06 \pm 1.37$ & - \\
\hline $\operatorname{OVIII}_{(\mathrm{Ly} \alpha) /(\operatorname{Ly} \gamma)}$ & 14.34 & $0.89 \pm 0.55$ & $1.54 \pm 1.17$ & - & - & - \\
\hline $\operatorname{OVIII}_{(\mathrm{Ly} \alpha) /(\operatorname{Ly} \delta)}$ & 29.92 & $0.71 \pm 0.41$ & $7.36 \pm 1.46$ & - & - & - \\
\hline $\operatorname{MgXI}_{(1.352) /(1.579)}$ & 4.84 & $1.15 \pm 0.19$ & - & - & - & - \\
\hline $\operatorname{MgXI}_{(1.352) /(1.660)}$ & 12.9 & $1.46 \pm 0.34$ & - & - & - & - \\
\hline $\operatorname{SiXIII}_{(1.865) /(2.182)}$ & 4.97 & $1.73 \pm 0.51$ & - & - & - & - \\
\hline $\operatorname{SiXIII}_{(1.865) /(2.294)}$ & 13.09 & $2.19 \pm 1.37$ & - & - & - & - \\
\hline $\operatorname{SiXIV}_{(\operatorname{Ly} \alpha) /(\operatorname{Ly} \gamma)}$ & 14.34 & $3.37 \pm 2.12$ & - & - & - & - \\
\hline $\operatorname{SXV}_{(2.461) /(2.883)}$ & 4.76 & $2.48 \pm 1.98$ & - & - & - & - \\
\hline $\operatorname{SXV}_{(2.461) /(3.032)}$ & 13.18 & $1.58 \pm 0.95$ & - & - & - & - \\
\hline $\operatorname{FeXVII}_{(0.8211) /(0.8984)}$ & 9.51 & $0.47 \pm 0.24$ & $0.88 \pm 0.99$ & - & - & - \\
\hline $\operatorname{FeXVII}_{(0.8211) /(1.008)}$ & 3.01 & $0.67 \pm 0.22$ & - & - & - & - \\
\hline $\operatorname{FeXXV}_{(6.700) /(7.881)}$ & 4.95 & $0.42 \pm 0.93$ & - & - & - & - \\
\hline
\end{tabular}

\section{Discussion}

We have calculated a grid of models of warm absorbers with density stratification determined by the condition of constant pressure. The assumption of constant pressure instead of constant density was motivated by recent observations of AGN. In most observed objects the ionization states implied by the observed lines span a large range, which cannot be accounted for a single photoionized region, or by collisionally ionized matter. At least two photoionized absorbing regions are required to fit the data and the properties of these two regions are consistent with pressure equilibrium

Our models represent an essential improvement in the description of a single cloud over the models presented in the literature. All previous models adopted a constant density approximation for the medium (Netzer 1993; Kaastra et al. 2002; Kinkhabwala et al. 2002; Krongold et al. 2005), or even constant temperature (Krolik \& Kriss 1995). On the other hand, we do not consider here a global picture including the dynamics of the warm absorber flow, which form a separate and major issue (see Chelouche \& Netzer 2005, and the references therein).

We have calculated the full radiative transfer of the illuminating radiation in continuum and in lines through plane-parallel density-stratified slabs of different total column densities. For a low total column density the cloud is mostly ionized and optically thin, and does not differ from the constant density model. For a higher total column density the optically thick dense zone arises at the back of the illuminated cloud, and the temperature falls dramatically. Therefore, absorbing matter contains zones of different ionization states coexisting under constant pressure.

For each set of models with spectral index $\Gamma$ and surface ionization parameter $\xi$ there is a maximum total column density $N_{\mathrm{H}_{\mathrm{tot}}}^{\mathrm{Max}}$ for which the cloud is thermally stable. For higher column densities thermal instabilities do not allow the calculations to converge. Interestingly, $N_{\mathrm{H}_{\mathrm{tot}}}^{\mathrm{Max}}$ is of the order of the maximum column density derived from X-ray observations of different AGN. It gives additional support to the idea of the warm absorber clouds being in pressure equilibrium.

The modeled lines are usually optically thick and their equivalent widths are of the order of the observed values when turbulent broadening is taken into account. We conclude that the observed warm absorbers at column densities of the order of $10^{22} \mathrm{~cm}^{-2}$ and higher possess saturated absorption lines and that full radiative transfer is required to model their equivalent widths properly. For full evaluation of this problem we will perform a curve of growth analysis in future work.

Generally lines are easier to detect than ionization edges and this tendency is observed in several AGN (Kaastra et al. 2002; Różańska et al. 2004). Our models predict that edges are not observable up to column densities about $10^{22} \mathrm{~cm}^{-2}$ and even higher, depending on $\Gamma$ and $\xi$.

The most interesting result of our computations is the shape of the spectrum around an iron $\mathrm{K} \alpha$ line. In almost all models there are strong and narrow absorption features due to highly ionized iron ions, above FeXVII. Such absorption affects the shape of the broad iron emission line possibly originating in the illuminated disk atmosphere. For instance, the relativistic broad iron line profile after passing through a warm absorber becomes disrupted into three narrower profiles, which can be fitted by a Gaussian. Such lines were reported in several AGN as presented by Yaqoob \& Padmanabhan (2004) (see also NGC 3783, Reeves et al. 2004).

In this paper we consider only a single cloud but observations suggest that absorbing material forms a distribution of clouds with a covering factor that may depend on the velocity of the clouds (de Kool et al. 2002). However, theoretical modeling of cloud distribution with a complex velocity field is complicated. Also, the study of the transmission spectra, including absorption lines, does not provide information on the matter in directions other than the line of sight. However, the TITAN code can be used 


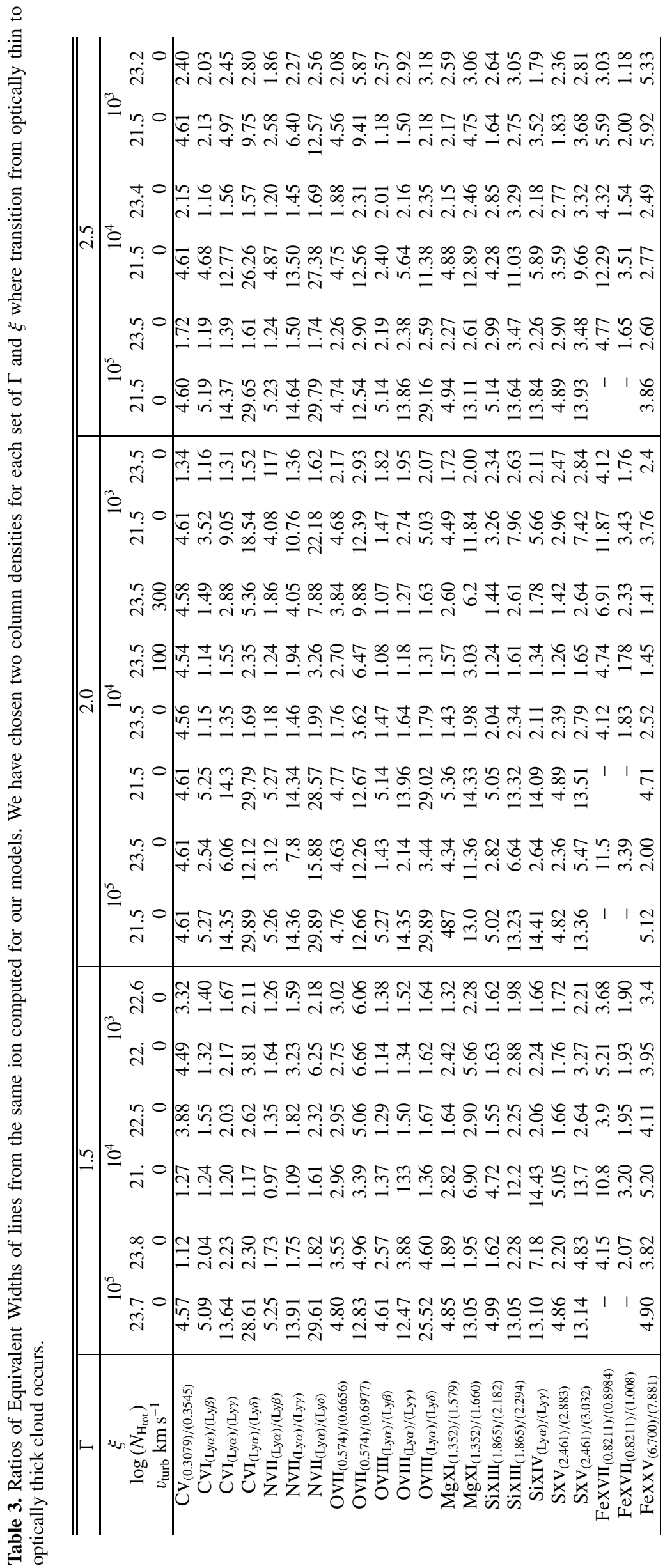


to study the radiative transfer in all directions. We will address both issues in future work.

Acknowledgements. We thank Suzy Collin, Martine Mouchet and Aneta Siemiginowska for helpful discussion. Part of this work was supported by grants 2P03D00322 and 1P03D00829 of the Polish State Committee for Scientific Research, the Laboratoire Européen Associé Astrophysique Pologne-France, and by the Hans-Böckler-Stiftung.

\section{References}

Ashton, A. C., Page, M. J., Branduardi-Raymont, G., \& Blustin, A. J. 2006 MNRAS, 366, 521

Antonucci, R. R. J., \& Miller, J. S. 1985, ApJ, 297, 621

Ballantyne, D., Weingartner, J. C., Murray, N., et al. 2003, A\&A, 409, 503

Barcons, X., Carrera, F. J., \& Ceballos, M. T. 2003, MNRAS, 346, 897

Bassani, L., Dadina, M., Maiolino, R., et al. 1999, ApJS, 121, 473

Behar, E., Rasmussen, A. P., Blustin, A. J., et al. 2003, ApJ, 598, 232

Blustin, A. J., Branduardi-Raymont, G., Behar, E., et al. 2003, A\&A, 403, 481

Blustin, A. J., Branduardi-Raymont, G., Behar, E., et al. 2005, A\&A, 431, 569

Chartas, G., Brandt, W. N., Gallagher, S. C., \& Garmire, G. P. 2002, ApJ, 279, 169

Chelouche, D., \& Netzer, H. 2005, ApJ, 625, 95

Collin, S., Dumont, A.-M., \& Godet, O. 2004, A\&A

Collinge, M. J., Brandt, W. N., Kaspi, S., et al. 2001, ApJ, 557, 2

Crenshaw, D. M., Kraemer, S. B., \& George, I. M. 2003a, ARA\&A, 41, 117

Crenshaw, D. M., Kraemer, S. B., Gabel, J. R., et al. 2003b, ApJ, 594, 116

Czerny, B., Różańska, A., Dovciak, M., Karas, V., \& Dumont, A.-M. 2004, A\&A, 419, 877

Dadina, M., \& Cappi, M. 2004, A\&A, 413, 921

Dasgupta, S., Rao, A. R., Dewangan, G. C., \& Agrawal, V. K. 2005, ApJ, 618, L87

de Kool, M., Korista, K. T., \& Arav, N. 2002, ApJ, 580, 54

Dumont, A.-M., Abrassart, A., \& Collin, S. 2000, A\&A, 357, 823

Dumont, A.-M., Collin S., Paletou F., et al. 2003, A\&A, 400, 437

Field, G. B. 1965, ApJ, 142, 531

Gabel, J. R., Kraemer, S. B., Crenshaw, D. M., et al. 2005, ApJ, 631, 74

George, I. M., Turner, T. J., Netzer, H., et al. 1998, ApJS, 114, 73

Guainazzi, M., Fiore, F., Matt, G., \& Perola, G. C. 2001, MNRAS, 327, 323

Halpern, J. P. 1984, ApJ, 281, 90

Kaastra, J. S., Steenbrugge, K. C., Raassen, A. J. J., et al. 2002, A\&A, 386, 427

Kaspi, S. 2004, in The Interplay among Black Holes, Stars and ISM in Galactic Nuclei, IAU

Symp., 222, ed. Th. Storchi Bergmann, L. C. Ho, \& H. R. Schmitt, 41

Kaspi, S., Brandt, W. N., Netzer, H., et al. 2001, ApJ, 554, 216

Kaspi, S., Brandt, W. N., George, I. M., et al. 2002, ApJ, 574, 643
Kaspi, S., Netzer, H., Chelouche, D., et al. 2004, ApJ, 611, 68

Kinkhabwala, A., Sako, M., Behar, E., et al. 2002, ApJ, 575, 732

Krolik, J. 2002, Workshop on X-ray Spectroscopy of AGN with Chandra and XMM-Newton, MPE Rep., 279, 131

Krolik, J., \& Kriss, G. A. 1995, ApJ, 447, 512

Krolik, J., \& Kriss, G. A. 2001, ApJ, 561, 684

Krolik, J. H., McKee, C. F., \& Tarter, C. B. 1981, ApJ, 249, 422

Krongold, Y., Nicastro, F., Brickhouse, N. S., et al. 2003, ApJ, 597, 832

Krongold, Y., Nicastro, F., Brickhouse, N. S., Elvis, M., \& Mathur, S. 2005, ApJ, 622, 842 Matt, G. 2000, A\&A, 327, L31

Matt, G., Bianchi, S., D’Ammando, F., \& Martocchia, A. 2004, A\&A, 421, 473

Mushotzky, R. F., Done, C., \& Pounds, K. A. 1993, ARA\&A, 31, 717

Nandra, K., \& Pounds, K. A. 1992, Nature, 359, 215

Nandra, K., \& Pounds, K. A. 1994, MNRAS, 268, 405

Netzer, H. 1993, ApJ, 411, 594

Netzer, H. 1996, ApJ, 473, 781

Netzer, H., Chelouche, D., George, I. M., et al. 2002, ApJ, 571, 256

Netzer, H., Kaspi, S., Behar, E., et al. 2003, ApJ, 599, 933

Otani, C., Kii, T., Reynolds, C. S., et al. 1996, PASJ, 48, 211

Piconcelli, E., Jimenez-Bailón, E., Guainazzi M., et al. 2004, MNRAS, 351, 161

Pounds, K. A., \& Page, K. L. 2005, MNRAS, 360, 1123

Pounds, K. A., King, A. R., Page, K. L., \& O'Brien, P. T. 2003a, MNRAS, 346, 102

Pounds, K. A., Reeves, J. N., King, A. R., et al. 2003b, MNRAS, 346, 1025

Reeves, J. N., Nandra, K., George, I. M., et al. 2004, ApJ, 602, 648

Reynolds, C. S. 1997, MNRAS, 286, 513

Reynolds, C. S., Fabian, A. C., Nandra, K., et al. 1995, MNRAS, 277, 901

Różańska, A., \& Czerny, B. 1996, Ac. A., 46, 233

Różańska, A., Dumont, A.-M., Czerny, B., \& Collin, S. 2002, MNRAS, 332, 799

Różańska, A., Czerny, B., Dumont, A.-M., Collin, S., \& Siemiginowska, A. 2004, ApJ, 600, 96

Scott, J. E., Kriss, G. A., Lee, J. C., et al. 2005, ApJ, 634, 193

Steenbrugge, K. C., Kaastra, J. S., Sako, M., et al. 2005a, A\&A, 432, 453

Steenbrugge, K. C., Kaastra, J. S., Crenshaw, D. M., et al. 2005b, A\&A, 434, 569

Schurch, N. J., Warwick, R. S., Griffiths, R. E., \& Kahn, S. M. 2004, MNRAS, 350, 1

Shih, D. C., Iwasawa, K., \& Fabian, A. C. 2003, MNRAS, 341, 973

Tanaka, Y., Nandra, K., Fabian, A. C., et al. 1995, Nature, 375, 659

Turner, A. K., Fabian, A. C., Lee, J. C., \& Vaughan, S. 2004, MNRAS, 353, 319

Turner, T. J., George, I. M., Nandra, K., \& Mushotzky, R. F. 1997, ApJS, 113, 23

Weaver, K. A., \& Reynolds, C. S. 1998, ApJ, 503, L39

Worsley, M. A., Fabian, A. C., Turner, A. K., Celotti, A., \& Iwasawa, K. 2004, MNRAS, 350,207

Yaqoob, T., \& Padmanabhan, U. 2004, ApJ, 604, 63

Yaqoob, T., McKernan, B., Kraemer, S. B., et al. 2003, ApJ, 582, 105 